This item was submitted to Loughborough's Research Repository by the author.

Items in Figshare are protected by copyright, with all rights reserved, unless otherwise indicated.

\title{
A memetic algorithm based on probability learning for solving the multidimensional knapsack problem
}

\section{PLEASE CITE THE PUBLISHED VERSION}

https://doi.org/10.1109/tcyb.2020.3002495

\section{PUBLISHER}

Institute of Electrical and Electronics Engineers (IEEE)

\section{VERSION}

AM (Accepted Manuscript)

\section{PUBLISHER STATEMENT}

(c) 2020 IEEE. Personal use of this material is permitted. Permission from IEEE must be obtained for all other uses, in any current or future media, including reprinting/republishing this material for advertising or promotional purposes, creating new collective works, for resale or redistribution to servers or lists, or reuse of any copyrighted component of this work in other works.

\section{LICENCE}

\section{All Rights Reserved}

\section{REPOSITORY RECORD}

Li, Zuocheng, Lixin Tang, and Jiyin Liu. 2020. "A Memetic Algorithm Based on Probability Learning for Solving the Multidimensional Knapsack Problem”. Loughborough University. https://hdl.handle.net/2134/14245553.v1. 


\title{
A Memetic Algorithm Based on Probability Learning for Solving the Multidimensional Knapsack Problem
}

\author{
Zuocheng Li, Lixin Tang Senior Member, IEEE, and Jiyin Liu
}

\begin{abstract}
The multidimensional knapsack problem (MKP) is a well-known combinatorial optimization problem with many real-life applications. In this paper, a memetic algorithm based on probability learning (MA/PL) is proposed to solve MKP. The main highlights of this paper are twofold: problem-dependent heuristics for MKP and a novel framework of MA/PL. For the problem-dependent heuristics, we first propose two kinds of logarithmic utility function (LUF) based on the special structure of MKP, in which the profit value and weight vector of each item are considered simultaneously. Then, LUFs are applied to effectively guide the repair operator for infeasible solutions and the local search operator. For the framework of MA/PL, we propose two problem-dependent probability distributions to extract the special knowledge of MKP, i.e., the marginal probability distribution (MPD) of each item and the joint probability distribution (JPD) of two conjoint items. Next, learning rules for MPD and JPD, which borrow ideas from competitive learning and binary Markov chain, are proposed. Thereafter, we generate MA/PL's offspring by integrating MPD and JPD, such that the univariate probability information of each item as well as the dependency of conjoint items can be sufficiently used. Results of experiments on 179 benchmark instances and a real-life case study demonstrate the effectiveness and practical values of the proposed MKP.
\end{abstract}

Index Terms - Combinatorial optimization, multidimensional knapsack problem, memetic algorithm, probability learning, competitive learning, Markov chain.

This research was partially supported by the Major International Joint Research Project of the National Natural Science Foundation of China (71520107004), the Fund for Innovative Research Groups of the National Natural Science Foundation of China (71621061), the Major Program of National Natural Science Foundation of China (71790614), the National Natural Science Foundation of China (71602025) and the 111 Project (B16009). (Corresponding author: Lixin Tang)

Z. Li is with Key Laboratory of Data Analytics and Optimization for Smart Industry (Northeastern University), Ministry of China, Shenyang, 110819, China (e-mail: zuocheng_li@163.com).

L. Tang is with Institute of Industrial \& Systems Engineering, Northeastern University, Shenyang, 110819, China (e-mail: lixintang@mail.neu.edu.cn).

J. Liu is with the School of Business and Economics, Loughborough University, Leicestershire LE11 3TU, U.K. (e-mail: j.y.liu@lboro.ac.uk).

\section{INTRODUCTION}

$\mathrm{T}$ HE MULTIDIMENSIONAL knapsack problem (MKP) is a generalized version of the conventional 0-1 knapsack problem. It is to select a subset of given items and put them into a multidimensional knapsack in order to maximize the total profit subject to the limited capacity of each dimension of the knapsack.

MKP is one of the most popular combinatorial optimization problems with rich practical applications, including cargo loading [1], cutting stock [2], portfolio selection [3], capital budgeting [4] and resource allocation in distributed systems $[5]$.

Let $\mathcal{N}$ be the set of given items, $\mathcal{N}=\{1, \ldots, N\}, \mathcal{M}$ the set of dimensions, $\mathcal{M}=\{1, \ldots, M\}, W_{j}$ the capacity of dimension $j \in \mathcal{M}, p_{i}$ the profit of item $i \in \mathcal{N}$, and $w_{i j}$ the weight of item $i$ in dimension $j$. Then, MKP can be formulated as follows:

$$
\begin{array}{ll}
\text { Max } & f=\sum_{i \in \mathcal{N}} p_{i} x_{i} \\
\text { Subject to } & \sum_{i \in \mathcal{N}} w_{i j} x_{i} \leq W_{j}, \forall j \in \mathcal{M}, \\
& x_{i} \in\{0,1\}, \forall i \in \mathcal{N},
\end{array}
$$

where $x_{i}$ defined in Constraints (3) is a $0-1$ decision variable indicating whether item $i$ is selected. The objective of the model (1) is to maximize the total profit. Constraints (2) ensure that the constraint of each dimension must be satisfied.

MKP is known to be NP-hard [6], and so the size of the problem that can be solved by exact algorithms [7]-[9] is limited. Even the latest exact algorithms (such as [9]) still have difficulties for instances with $N=250$ and $M=30$. In this case, metaheuristics can be suitable solution methods because they can obtain near optimal solutions within acceptable running time [10]. Memetic algorithm (MA) is a metaheuristic which uses a hybrid search model integrating evolutionary algorithms [11]-[12] and local search to achieve a good balance between the global exploration and the local exploitation. It has been proved that MAs are effective for many combinatorial optimization problems [13]-[16] and in particular for MKP [17]-[19]. This motivates our MA search framework for MKP.

Learning-based mechanisms have been widely advocated in enhancing the search performances of MAs [20]-[22]. For most learning-based MAs, certain kinds of probability models are adopted to extract problem-specific knowledge and generate 
offspring. As Gupta and Ong [23] pointed out, accurate probability models are useful for improving the global exploration of MAs. In this sense, suitable probability models are critical to learning-based MAs, which in general can be represented in different forms, such as a probability distribution or other learning-based networks [24], depending on the problem being solved. The above works motivate us to propose an MA based on probability learning (MA/PL) to solve MKP, in which a problem-specific probability model is introduced.

There are various kinds of probability models in existing learning-based metaheuristics, such as estimation of distribution algorithm (EDA) [25]-[28], cross-entropy algorithm (CEA) [29] and ant colony optimization (ACO) [30]. In MA/PL, a simple probability model (i.e., probability distribution) is used. The probability model of MA/PL is different from EDA, CEA and ACO in that it considers the problem-specific knowledge of MKP. Based on the structures of MKP, the probability model of MA/PL can reveal the inherent relationships among items. We not only consider individual items, but also the dependency of two conjoint items. Two kinds of probability distribution are used in MA/PL: 1) the marginal probability distribution (MPD) for each item to be selected; 2) the joint probability distribution (JPD) for two conjoint items to be selected simultaneously. Whereas MPD reflects the univariate relationships of items, JPD reflects the connections of conjoint items. Complementing each other, MPD and JPD work together to improve the global exploration ability of MA/PL. Very recently, Cai et al. [31]-[32] proposed a novel dual population framework which used an external archive (ExA) to store elite solutions found during the search process. It can make good use of the information from ExA for generating high-quality solutions. In MA/PL, we introduce an ExA as well, which is used to build MPD and JPD.

Infeasible solutions might be generated during the search process of MA/PL. In this case, introducing suitable constraint handling techniques is crucial for the search performance [33]. In existing works, the concept of utility has been shown useful for designing repair operators for MKP. In [34]-[35], two novel utility-based repair operators for MKP, i.e., RO2 and RO3, were proposed. $\mathrm{RO} 2$ and $\mathrm{RO} 3$ are guided by the pseudo-utility ratio of each item that is related to the objective function and the constraint violation. It is not uncommon, however, that the values of the objective function and the constraint violation are in different magnitude. Thus, normalization is necessary. In this paper, we advance existing utility-based repair operators and embed the logarithmic utility function (LUF) [36] into the proposed repair operator. LUF can obtain a composite value by weighting the preference scales of the objective function and the constraint violation. Thereby, we propose an LUF-based repair operator (RO_LUF). RO_LUF dynamically considers the profits of items and their contributions to the current overload of each dimension, so it is different from $\mathrm{RO} 2$ and RO3.

Local search is another important component of MAs. Given any feasible solution of MKP, local search can be viewed as an inverse process of the repair operator and so may be guided by an LUF similar to that used in the repair operator.
We propose a simple yet effective local search based on LUF (LS_LUF), where neighboring solutions are generated according to another LUF that considers the profits of the items and spare capacity they need in each dimension. Such a problem-specific LS_LUF intends to enhance the local exploitation ability of MA/PL.

The framework of the proposed MA/PL is given in Fig. 1. As shown in Fig. 1, it starts with a randomly generated initial population. The individuals in the population are repaired and improved using RO_LUF and LS_LUF and then the best ones among them are selected to form the initial ExA. Next, the probability models MPD and JPD are built by using ExA, and used to generate a population of the next generation. The individuals in the new population are repaired and improved, the resulting feasible individuals are used to update ExA, and the iteration continues until some stopping criteria are satisfied.

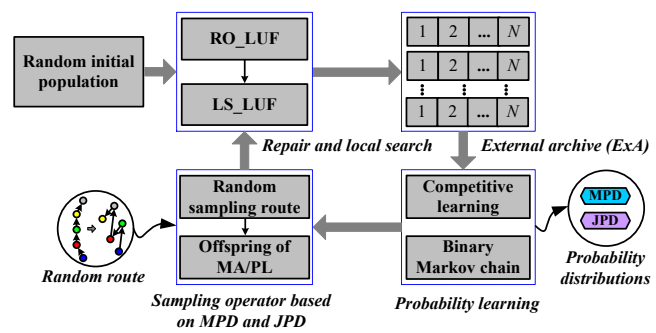

Fig. 1. Sketch of the proposed MA/PL for MKP

We summarize the contributions of this paper as follows.

1) Based on features of MKP, we propose two LUFs considering the profit of each item and the overload/remaining capacity of each dimension. The LUFs are used to measure the tradeoff between the objective and the capacity constraint when performing the repair operation and the local search operation.

2) We propose the probability learning rules for MPD and JPD, which are derived from the competitive learning and the binary Markov chain. Especially JPD considers the dependency of two conjoint items in generating solutions of MKP. Integrating MPD and JPD strengthens the global search of $\mathrm{MA} / \mathrm{PL}$.

3) We introduce a random route in MA/PL's sampling operator, such that the overall dependency of items can be potentially revealed based on the dependency of two conjoint items.

The rest of the paper is organized as follows. Section II introduces related work on MA and MKP. In Section III, based on the solution representation of MKP, the proposed RO_LUF and LS_LUF are described. Section IV presents MA/PL in detail. Results of experiments on 179 benchmark instances and a real-life case study are presented in Sections V and VI. Finally, Section VII draws conclusions and proposes future work.

\section{RELATED WORK}

MAs have been successfully adopted to solve various optimization problems. A comprehensive review of MAs can be found in Chen et al. [37]. MA is naturally suitable for combi- 
natorial optimization problems, due to the fact that local search can provide basic building blocks in discrete search domains. There have been only a few studies that use MA to solve MKP. Özcan and Başaran [17] proposed a genetic algorithm (GA) with hill climbing heuristic. Rezoug et al. [18] proposed two GA-based MAs which incorporated a stochastic local search and a simulated annealing. Sörensen and Sevaux [19] reported an MA with a population management strategy. These works show that MAs can achieve good performance for MKP and so motivate the proposed MA-based search model.

Machine learning has shown powerful ability for enhancing the search performance of MAs. Ong and Keane [21] proposed a Meta-Lamarckian learning to improve the local search of MAs. Rakshit et al. [22] reported an adaptive MA based on Q-Learning for multi-robot path planning. Indeed, the foremost highlight of learning-based MAs is the probability model. Different kinds of probability models have been proposed in previous learning-based metaheuristics. Liang et al. [25] proposed an EDA with a Gaussian probability model for the function optimization. In [26]-[27], EDAs with Gaussian probability models were used to solve multimodal problems. Shim et al. [28] integrated an underlying EDA into the multi-objective search model. The above works show that suitable probability models should reflect the features of problems studied. These also motivate the proposed probability models of MA/PL.

Research on MKP started in the 1950s, from seminal works of Lorie and Savage [38], Markowitz and Manne [39], Loulou and Michaelides [40], and Magazine and Oguz [41]-[42]. In [43], a comprehensive review of studies on MKP is provided. Generally, solution methods for MKP can be classified as optimization-based algorithms and metaheuristics.

There are a number of optimization-based algorithms proposed for solving MKP which apply optimization methods as part of the solution process. Hill et al. [44] proposed a problem reduction heuristic for MKP, where they first formulated MKP as a Lagrangian dual problem and then presented a heuristic for solving the estimated core problem. Yoon et al. [45] reported a Lagrangian method using the concept of Lagrangian capacity/relaxation for MKP. Rong et al. [46] solved a kind of multicriteria MKP with $k$-min objectives using hybrid algorithms. Other optimization-based methods, such as branch and bound [1], [7] and collaborative methods [8], were also proposed to solve MKP. Most of the above algorithms can only get optimal solutions for instances of small or medium sizes. Although some of them can obtain near optimal solutions for large-sized ones, such as [8], [44]-[45], the computation time may increase dramatically when the scale of instances increases.

Metaheuristics are more suitable for solving large scale MKP. Chih [47] reported a particle swarm optimization (PSO) with a self-adaptive check and a repair operator (SACRO) for MKP. Zhang et al. [48] solved MKP using a binary artificial algae algorithm (BAAA) derived from the artificial algae algorithm. Zouache et al. [49] presented a quantum-inspired firefly algorithm with PSO (QIFAPSO) for MKP. López et al. [50] proposed a hybrid algorithm with a binary PSO and ge- netic operators (HPSOGO) for MKP. Bhattacharjee and Sarmah [51] proposed two metaheuristics for MKP, i.e., BCSA and BFA, based on cuckoo search and firefly algorithm in continuous domains. Other metaheuristics were also used to solve MKP, such as binary differential search (TR-BDS) [52], binary harmony search (NBHS2) [53], $k$-means transition ranking cuckoo search (KMTR-Cuckoo) and black hole (KMTR-BH) [54], hybrid binary differential evolution with RO3 (HBDE-RO3) [35], modified multi-verse optimization (MMVO) [60] and teaching-learning based optimization (TLBO) [61]. The above papers showed advantages of metaheuristics for MKP.

However, for MKP, studies that incorporate machine learning into metaheuristics are relatively unexplored. Wang et al. [34] presented a hybrid EDA (HEDA) for MKP, which adopted a probability matrix to record the distribution of elite solutions. Arin and Rabadi [55] proposed a randomized priority search model (Meta-RaPS) for MKP, whereby presented a Meta-RaPS EDA and a Meta-RaPS Q by integrating EDA and Q-learning into Meta-RaPS. Rojas-Morales et al. [56] presented an ant knapsack algorithm based on opposite learning for MKP. The above works show that there is still a gap between machine learning and metaheuristics for solving MKP.

\section{Proposed ProbleM-DEPENDENT HeURISTICS}

In this section, we describe the solution representation and the problem-dependent heuristics, i.e., RO_LUF and LS_LUF that are used as repair and local search operators in MA/PL.

\section{A. Solution Representation}

In MA/PL, a string of $N$ bits is used to represent a solution. Value 1 of a bit represents that the item is selected while value 0 means not selected. Given any solution, it may or may not be feasible. If infeasible, we can repair it by taking some items out. For feasible solutions we can try to improve it by adding more items in. In both operations, we propose to choose items based on LUFs. The idea of LUF stems from the utility concept in quality management areas [36]. We define an LUF as a metric that measures the effect of a given item on the total profit and constraints, and use LUFs to determine the priority of candidate items in RO_LUF and LS_LUF. Fig. 2 shows an infeasible solution $\pi$ and candidate items in it for RO LUF, as well as a feasible solution $\boldsymbol{\pi}^{\prime}$ and candidate items in it for LS_LUF.

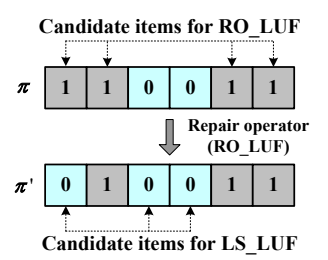

Fig. 2. Illustration of candidate items for RO_LUF and LS_LUF 


\section{B. Proposed Repair Operator Based on LUF (RO_LUF)}

The repair operator for infeasible solutions can be seen as a readjustment of the load in each dimension. It is necessary to consider the overload in each dimension. Let $\boldsymbol{o l}=\left[o l_{1}, \ldots, o l_{M}\right]$ be the overloads in the $M$ dimensions for an infeasible solution $\boldsymbol{\pi}=\left[\pi_{1}, \ldots, \pi_{N}\right]$. Each element of $\boldsymbol{o} \boldsymbol{l}$ can be calculated as

$$
o l_{j}=\sum_{i \in \mathcal{N}} w_{i j} \pi_{i}-W_{j}, j \in \mathcal{M},
$$

where the first term is the load in dimension $j$. Obviously, a negative or zero value of $o l_{j}$ indicates that the capacity constraint in dimension $j$ is satisfied.

In case $o l_{j}$ is positive for some $j$, the solution is infeasible. To repair the solution, we take out items one by one until the solution becomes feasible. Denote $\mathrm{Cr}$ as the set of candidate items to be taken out (for example, $\mathrm{Cr}=\{1,2,5,6\}$ in Fig. 2). To determine the priority of the candidate items, we calculate an LUF value for each item $i \in C r$ using two attributes of the item. One is its profit $p_{i}$, another reflects its impact on the capacities in all dimensions, which is defined as

$$
\psi_{i}=\sum_{j \in \mathcal{M}} \max \left\{\varepsilon, o l_{j}-w_{i j}\right\}, \forall i \in C r,
$$

where we set $\varepsilon=0.001$ to ensure $\psi_{i}>0$. Then, the value of LUF in RO_LUF is defined as

$$
\sigma_{i}=\lambda_{1} \cdot \log \left(\max _{i \in C r}\left\{p_{i}\right\} / p_{i}\right)+\lambda_{2} \cdot \log \left(\max _{i \in C r}\left\{\psi_{i}\right\} / \psi_{i}\right),
$$

where $\lambda_{1}$ and $\lambda_{2}$ are coefficients. Without loss of generality, we set $\lambda_{1}=\lambda_{2}=0.5$ to represent the equal importance of the two attributes for given items. Obviously, when changing the bits of items in $C r$ from 1 to 0 , an item that has larger LUF will result in smaller decrease of total profit while making more contribution in getting the capacities satisfied.

In RO LUF, the item in $\mathrm{Cr}$ with the largest LUF is taken out, the related bit is changed from 1 to 0 , and this item is taken out of $C r$. If the solution is still infeasible, the values of $\psi_{i}$ and $\sigma_{i}$ are recalculated to identify the next item to be taken out. This process continues until the solution becomes feasible. The proposed RO_LUF is given in Algorithm 1.

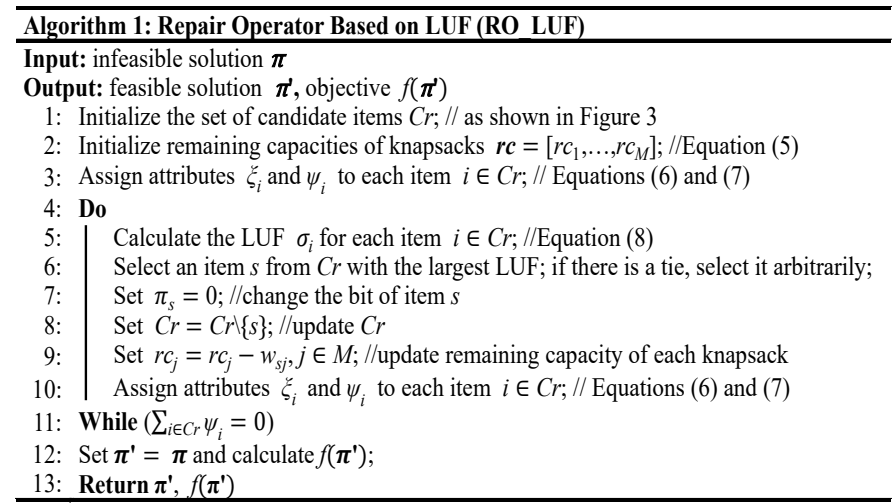

In the illustration example in Fig. 2, RO_LUF is applied on the infeasible solution $\pi$. After one iteration taking out item 1 that has the largest LUF, a feasible solution $\boldsymbol{\pi}^{\prime}$ is obtained.

\section{Proposed Local Search Based on LUF (LS_LUF)}

As an inverse process of the repair operator, we here consider the spare capacity in each dimension. For a feasible solution $\boldsymbol{\pi}^{\prime}=\left[\boldsymbol{\pi}_{1}^{\prime}, \ldots, \pi_{N}^{\prime}\right]$, let $\boldsymbol{s} \boldsymbol{c}=\left[s c_{1}, \ldots, s c_{M}\right]$ be the spare capacities in the $M$ dimensions. Each element can be calculated as

$$
s c_{j}=W_{j}-\sum_{i \in \mathcal{N}} w_{i j} \pi_{i}^{\prime}, \forall j \in \mathcal{M} .
$$

Let $\mathrm{Cl}$ be the set of items not in the knapsack (for example, $C l=\{1,3,4\}$ in Fig. 2), such that adding each of them alone does not result in capacity violation

$$
C l=\left\{i \mid \pi_{i}^{\prime}=0 \wedge s c_{j}-w_{i j} \geq 0, \forall i \in \mathcal{N} \text { for all } j \in \mathcal{M}\right\} .
$$

We calculate another LUF for item $i \in \mathrm{Cl}$, again using two attributes, the profit $p_{i}$ and a specially defined $\tau_{i}$

$$
\tau_{i}=\sum_{j \in \mathcal{M}}\left(s c_{j}-w_{i j}\right)+\varepsilon, i \in C l,
$$

where $\varepsilon=0.001$ is used to guarantee $\tau_{i}>0$. Then, this LUF for item $i \in C l$ is calculated as

$$
\delta_{i}=\lambda_{1} \cdot \log \left(\max _{i \in C l}\left\{p_{i}\right\} / p_{i}\right)+\lambda_{2} \cdot \log \left(\max _{i \in C l}\left\{\tau_{i}\right\} / \tau_{i}\right),
$$

where $\lambda_{1}=\lambda_{2}=0.5$ are two coefficients. In LS_LUF, we identify the item with the smallest LUF and change its bit from 0 to 1.

LS_LUF is also an iterative procedure, each time choosing one item to add in, then recalculating spare capacity of each dimension, updating $\mathrm{Cl}$, and recalculating $\tau_{i}$ and $\delta_{i}$, until $\mathrm{Cl}$ is empty. This procedure is given in Algorithm 2.

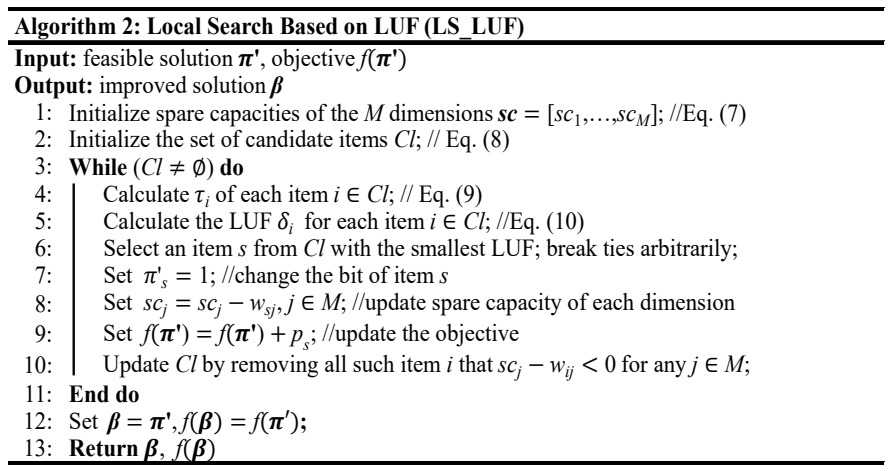

Note that we use LS_LUF to improve only the feasible solutions that are generated by repairing infeasible solutions with RO_LUF, such as $\boldsymbol{\pi}^{\prime}$ generated by repairing $\boldsymbol{\pi}$ in Fig. 2. This is because, in our preliminary tests, we observed that executing LS_LUF for all feasible solutions produced by MA/PL's global search is very likely to cause a premature convergence.

\section{MA/PL FOR MKP}

In MP/PL, we use two learning rules: 1) the learning rule based on the competitive learning for MPD; 2) the learning rule based on the proposed binary Markov chain for JPD. Using the learning rules, the dependency of items can be captured when a new population is produced by using a random sampling route. 


\section{A. ExA and Updating Mechanism}

ExA is updated at each generation of MA/PL, so that it always contains high-quality solutions. Let $P S$ be the population size of MA/PL, $A L$ the size of ExA, $\boldsymbol{\pi}^{\boldsymbol{P}}($ gen $)=\left\{\boldsymbol{\pi}_{1}^{p}(\right.$ gen $), \ldots$, $\boldsymbol{\pi}_{P S}^{p}($ gen $\left.)\right\}$ the population of MA/PL at generation gen, and $\boldsymbol{\pi}^{e}(\mathrm{gen})=\left\{\boldsymbol{\pi}_{1}^{e}(\mathrm{gen}), \ldots, \boldsymbol{\pi}_{A L}^{e}(\mathrm{gen})\right\}$ the set of ExA at generation gen. ExA can be initialized and updated by Algorithm 3.

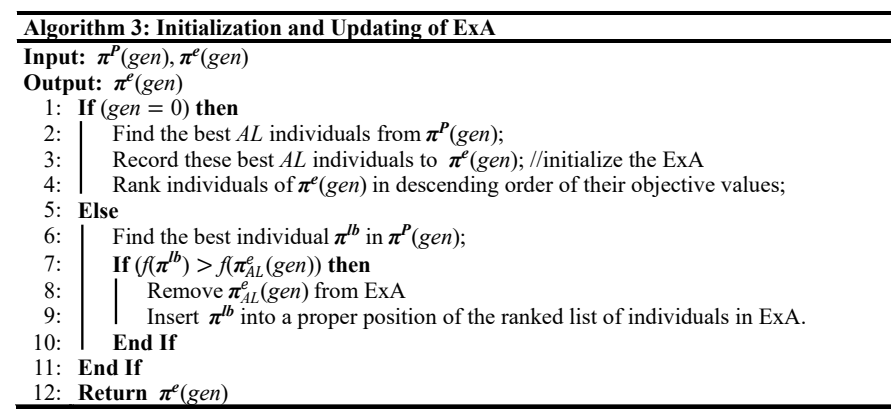

In Algorithm 3, ExA is incrementally updated by replacing the worst individual in it with the best in the current population if the latter is better. Thus, the information of ExA that reflects elite solutions can be gradually enriched. No more than one individual from the current population is added to ExA to avoid premature convergence. MPD and JPD are all based on ExA, so it can be seen as a set of training data for our learning rules.

\section{B. Learning Rule Based on Competitive Learning for MPD}

The ideas of the proposed learning rule for MPD derive from competitive learning [57]. The competitive learning can make good use of the information of high-quality samples in learning probability models. We represent MPD as the union of the univariate marginal distributions of all items. Then the existing learning rule in [34] and [57] is used. However, we learn MPD from ExA rather than from elite individuals in current population, which is different from [34]. Let $\boldsymbol{M P r}$ (gen) be the union of the marginal distribution at generation gen. It is defined as

$\operatorname{MPr}($ gen $)=\left[\begin{array}{l}m p r_{0,1}\left(\text { gen } \ldots_{\ldots} \ldots m r_{0, i}(\text { gen }) \ldots m p r_{0, N}(\text { gen })\right. \\ m p r_{1,1}(\text { gen }) \cdots m p r_{1, i}(\text { gen }) \cdots m p r_{1, N}(\text { gen })\end{array}\right]_{2 \times N}$,

where $\sum_{k=0}^{1} \mathrm{mpr}_{k, i}($ gen $)=1(i \in \mathcal{N})$ and $m p r_{k, i}($ gen $)$ represents the probability value for the bit of item $i$ to be $k$. Note that $\operatorname{MPr}($ gen $)$ is initialized with $m p r_{k, i}($ gen $=0)=0.5(i \in \mathcal{N}$ and $k$ $\in\{0,1\}$. Let $L R$ be the learning rate of MA/PL. The competitive learning method for estimating MPD is given in Algorithm 4.

From Algorithm 4, it can be seen that MPDs between two consecutive generations are connected with the learning rate $L R$, and the probability values of items are gradually moved towards TmpPro/AL obtained from elite solutions (Line 6). This is similar to the weight update rule in competitive learning. In this case, MPD is not only determined by its previous statuses, but also related to the information of the current ExA (Lines 3 to 6). Thus, the information of elite solutions can be adequately used by MA/PL, so as to predict promising search regions.
Algorithm 4: Learning Rule based on Competitive Learning for MPD

Input: $\pi^{e}($ gen -1$), \operatorname{MPr}($ gen -1$)$

Output: $\operatorname{MPr}($ gen $)$

1: For $i=1$ to $N$ do

1: $\quad$ For $i=1$ to $N$ do
2: $\quad$ Set TmpPro $=0$

3: $\quad$ For $k=1$ to $A L$ do

4: I Set TmpPro=TmpPro $+\boldsymbol{\pi}_{k, i}^{e}($ gen -1$) ; / /$ calculate the total number of bit 1

5: $\quad$ End For

6: $\quad$ Set $m p r_{1, i}($ gen $)=(1-L R) \cdot m p r_{1, i}($ gen -1$)+L R \cdot T m p P r o / A L ; / /$ competitive learning

7: $\quad$ Set $m p r_{0, j}$ (gen $)=1-m p r_{1, j}$ (gen); //competitive learning

8: End For

9: Return $\operatorname{MPr}($ gen $)$

\section{Learning Rule Based on Binary Markov Chain for JPD}

The purpose of JPD is to get the dependency of two conjoint items. Our learning rule for JPD is motivated from the binary signal source in telegraph systems [58]. The telegraph system outputs a series of pulses with high $(\mathrm{H})$ and low $(\mathrm{L})$ symbols (i.e., an $N$-bit string) in a temporal order. It can be modeled by a Markov source with given memory length. The memory length defines the dependency structure of signals. If we only consider a dependency of two conjoint symbols, the memory length is 1 (i.e., first-order Markov source). The first-order Markov source can be analyzed by a binary Markov chain. As shown in Fig. 3, the dependency of two conjoint symbols can be captured using the binary Markov chain, such as the $(i-1)$ th and the $i$ th signals.

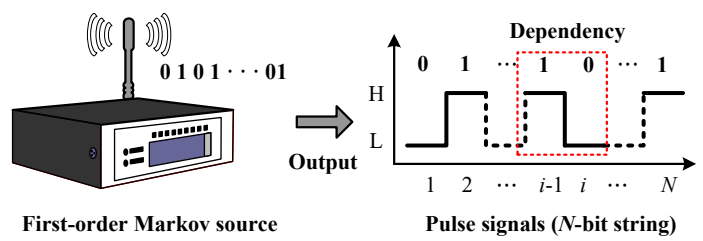

Fig. 3. First-order Markov source and dependency of symbols

It is interesting that the output of the first-order Markov source is equivalent to the solution representation of MKP (see Fig. 4), when regarding the "temporal order" as the "spatial order". Thus, it is reasonable to extract the bivariate dependency of conjoint items by using a binary Markov chain.

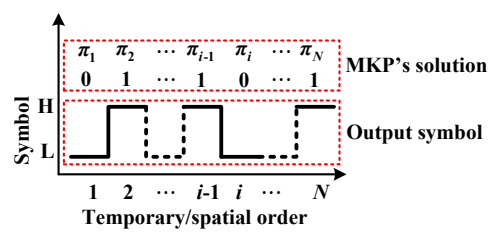

Fig. 4. Output of Markov source and MKP's solution

In Fig. 4, the probability distributions of points 2 to $N$ are often different, which are determined by the features of MKP. Therefore, the non-stationary binary Markov chain is used in our learning rule, as shown in Fig. 5. There are $N-1$ pairs of conjoint items for the order $2 \rightarrow \ldots \rightarrow N$. For each point of 2 to $N$, we first calculate the one-step transition probability matrix (OSTPM) from ExA and in turn obtain JPD based on OSTPM.

Let pro $_{i, \rho}(i \in\{2, \ldots, N\}$ and $\rho \in\{1, \ldots, 4\})$ be the OSTPM for the transitions of states on the $i$ th point, where pro $_{i, 1}:(0 \rightarrow 0)$, pro $_{i, 2}:(0 \rightarrow 1)$, pro $_{i, 3}:(1 \rightarrow 1)$ and pro $i, 4:(1 \rightarrow 0)$. Then, OSTPM for the $i$ th point can be calculated using Algorithm 5 . 


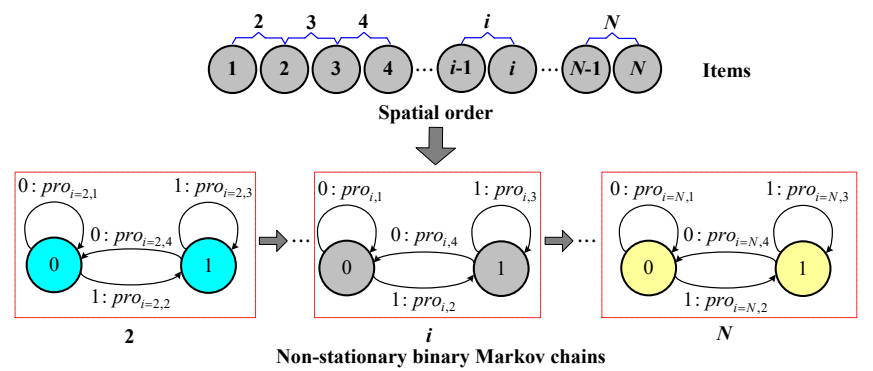

Fig. 5. Spatial order and non-stationary binary Markov chain ( $N$ items)

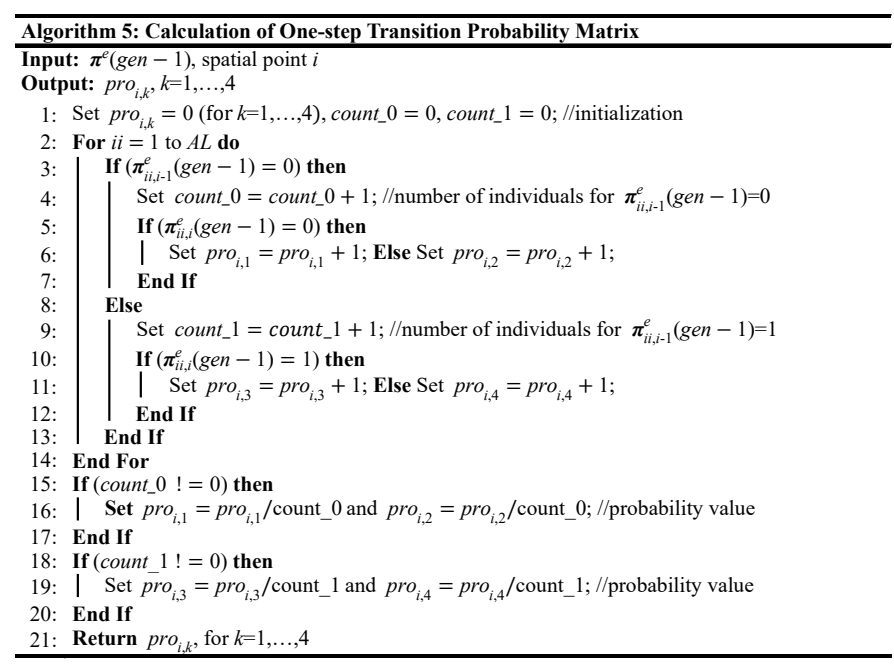

Based on OSTPM, one can calculate JPD for each spatial point $i \in\{2, \ldots, N\}$. Let $\boldsymbol{J P r}^{i}($ gen $)=\left[j p r_{k, l}^{i}(\mathrm{gen})\right]_{2 \times 2}$ be the JPD for spatial point $i$ at generation gen. Then, it satisfies

$$
\sum_{k \in\{0,1\}} \sum_{l \in\{0,1\}} j p r_{k, l}^{i}(\text { gen })=1,
$$

where $j p r_{k, l}^{i}(\mathrm{gen})$ is the probability of $\pi_{i-1}=k$ and $\pi_{i}=l$ at generation gen. Apparently, $\boldsymbol{J P r}^{i}($ gen $)$ can be seen as a discrete joint distribution of conjoint items $i-1$ and $i$ in the order of $2 \rightarrow \ldots \rightarrow N$. Then, we can calculate $j p r_{k, l}^{i}($ gen $)$ as

$$
j p r_{k, l}^{i}(\text { gen })=\left\{\begin{array}{l}
\left(\frac{1}{A L} \cdot\left(A L-\sum_{i \in \in\{1, A L\}} \pi_{i, i, i-1}^{e}(\text { gen }-1)\right)\right) \cdot \text { pro }_{i, \rho} \quad k=0 \\
\left(\frac{1}{A L} \cdot \sum_{i i \in\{1, A L\}} \pi_{i, i,-1}^{e}(\text { gen }-1)\right) \cdot \text { pro }_{i, \rho} \quad \text { otherwise }
\end{array}\right.
$$

where $\rho$ is set to $1,2,3$ and 4 for conditions $(k=0) \wedge(l=0),(k=$ $0) \wedge(l=1),(k=1) \wedge(l=1)$ and $(k=1) \wedge(l=0)$ respectively.

\section{Sampling Operator Based on MPD and JPD}

Accurate probability models are beneficial to improve the global exploration of learning-based MAs [23]. Indeed, there are many probability models that can capture the precise dependency of all random variables, such as Bayesian networks. However, such probability models in general take more computation time. Hence, we only consider the dependency of two conjoint items in the proposed JPD. Besides, there may be a dependency between any pair of items. In this case, many such dependencies would not be considered if one fixed sampling route is always used. Thus, we propose to take a random sampling route, so that the dependency between any items can be potentially considered. In our sampling operator, we first generate a random permutation of integers $1, \ldots, N: \boldsymbol{r}=\left[r_{1}, \ldots, r_{N}\right]$ and then use it as the sampling route, as shown in Fig. 6.

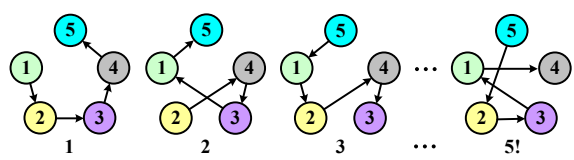

Fig. 6. Illustration of sampling route $(N=5)$

Let $\operatorname{cpro}\left(\boldsymbol{\pi}_{r i}=l \mid \boldsymbol{\pi}_{r i-1}=k\right), i \in\{2, \ldots, N\}$ be the conditional probability of $\boldsymbol{\pi}_{r i}=l$ given $\boldsymbol{\pi}_{r i-1}=k$ in terms of sampling route $\boldsymbol{r}$. We can calculate it via the conditional probability formula as

$$
\text { cpro }\left(\pi_{r i}=l \mid \pi_{r i-1}=k\right)=j p r_{k, l}^{r_{i}}(\text { gen }) / \sum_{k \in\{0,1\}} j p r_{k, l}^{r_{i}}(\text { gen }) \text {. }
$$

Afterwards, the offspring can be produced by integrating MPD and JPD. The sampling operator is given in Algorithm 6 .

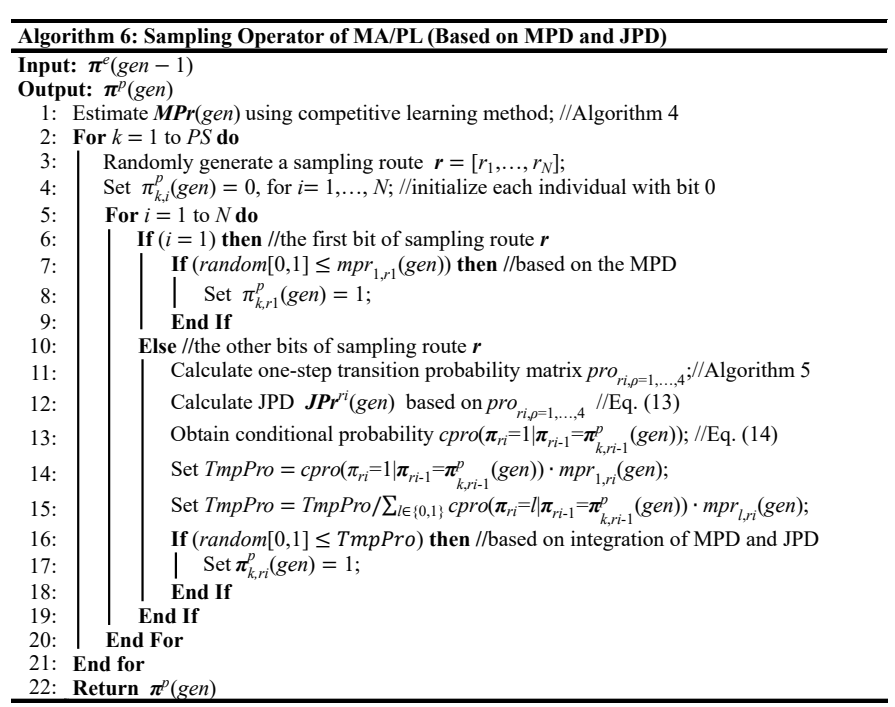

In Algorithm 6, a sampling route is first generated (Line 3), which is helpful in capturing more potential dependency among items. Then, the first bit of the sampling route is generated from MPD (Lines 6 to 11), while other bits are generated by integrating JPD and MPD (Lines 11 to 20). Thus, MPD and JPD can complement each other in the global search of MA/PL.

\section{E. Overall Procedure of Proposed MA/PL}

Let genMax be the maximum generations and gbest(gen) the best feasible individual found so far. The overall procedure of $\mathrm{MA} / \mathrm{PL}$ is given in Algorithm 7. It shows that MPD and JPD are all estimated from ExA (Lines 5 and 10), which is useful to get more information of promising search regions. Moreover, we use RO_LUF and LS_LUF to repair and improve new solutions (Lines 4 and 9). With this precise model, MA/PL can hopefully achieve a good balance between exploration and exploitation.

\section{F. Time Complexity of Proposed MA/PL}

The time complexities of calculating the objective function, initializing population and sampling new individuals are all 
$O(P S \cdot N)$. In the worst case, the time complexities of RO LUF and LS_LUF are all $O(P S \cdot N \cdot(3 \cdot N+M))$. The time complexities of initializing and updating ExA are all $O(A L)$. The time complexity of learning and initializing MPD are all $O(N)$. The time complexity of learning JPD is $O((N-1) \cdot A L+N)$. Thus, the total time complexity is genMax $\cdot\left\{O\left(P S \cdot N^{2}+P S \cdot N \cdot M\right)+O(N \cdot A L)\right\}$.

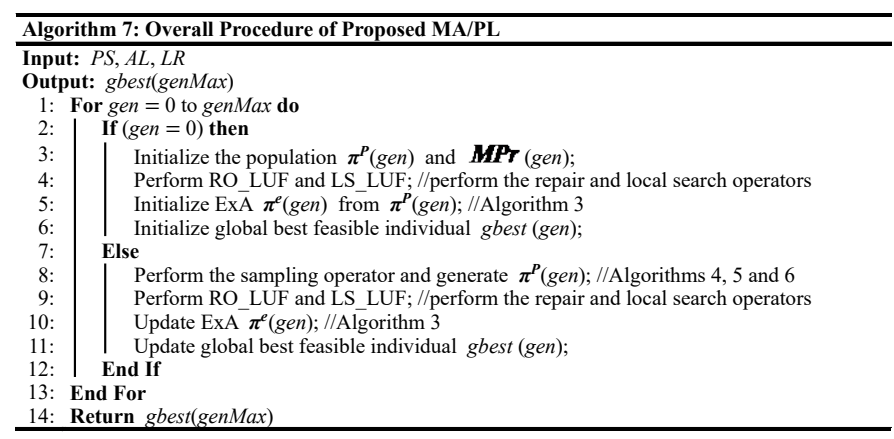

\section{EXPERIMENTAL RESULTS AND COMPARISONS}

\section{A. Experimental Setup and Parameter Settings}

To verify the performance of the proposed MA/PL, we test it on four sets of well-known benchmark data for MKP. The sets are related to different sizes. Test set I contains 11 large scale instances with $M=15$ to 100 and $N=100$ to 2500 (available from http://hces.bus.olemiss.edu/tools.html). Test set II contains 20 medium scale instances with $M=5$ and 10 and $N=100$ (available from ORLIB [59]). Test set III contains 58 small scale instances from $M=2$ to 30 and $N=10$ to 105 (available from ORLIB). Test set IV consists of 90 large scale instances with $M=5$ to 30 and $N=500$ (available from ORLIB). Therefore, there are a total of 179 instances. MA/PL is coded in $\mathrm{C}++$ and run on a PC with Intel Core-i7 $3.4 \mathrm{GHz}$ processor and $16 \mathrm{~GB}$ memory.

$\mathrm{MA} / \mathrm{PL}$ is independently run 30 times for each instance. Results are reported with 8 metrics, i.e., $B E S T, A V G, S D, S R$, Min.Dev, Ave.Dev, Max.Dev and Var.Dev. BEST, AVG, SD and $S R$ are the best value, mean value, standard deviation and success rate. Min.Dev, Ave.Dev and Max.Dev are the minimum, average and maximum percentage deviations from best-known solutions (BKS). Var.Dev is the variance of percentage deviations. We note that the values of BKS for all the instances are collected from the most currently published works for MKP (available at http://dx.doi.org/10.13140/RG.2.2.14761.95841). In Tables I to XIV, each number in the row \#Best is the total number of instances for which the relevant algorithm obtained the best result in terms of different quality metrics. Besides, we highlight the best quality metric of each instance found by relevant algorithms in bold, and mark the column of BEST of certain instances with symbol "**" if BKS is attained. To check the significance of the differences between MA/PL and other algorithms, the $p$-values based on the non-parametric Friedman test at the $95 \%$ confidence level (CI) are reported as well.

In subsections V-C and V-D we adopt the results of existing algorithms from relevant references. The average CPU times (s) (i.e., CPU (s)) of MA/PL are listed for information. Due to the differences in programming environments and computer con- figurations, it is hard to accurately compare CPU times of other algorithms. However, we find that CPU times of MA/PL are all acceptable and thus running times will not be further discussed.

The parameters of MA/PL include the population size $P S$, the size of ExA $A L$ and the learning rate $L R$. To obtain the appropriate parameters, relevant experiments based on the design-of-experiment (DOE) are carried out. We set the parameters of MA/PL as follows: $P S=150, A L=20$ and $L R=0.01$. More details about the parameter settings of MA/PL can be found at http://dx.doi.org/10.13140/RG.2.2.23150.56644.

\section{B. Effectiveness of MA/PL's Components}

\section{1) Effectiveness of proposed learning rules and local search}

To study the impact of the proposed learning rules, random sampling route and local search, we introduce the following variants. 1) MA/PL V1: it is different from MA/PL in that the learning method for JPD, the random sampling route and LS_LUF are removed. That is, MA/PL_V1's search behavior is only guided by the learning and sampling operators of MPD. 2) MA/PL_V2: it is the same as MA/PL_V1 except that the learning method for JPD is added. 3) MA/PL_V3: it is obtained by replacing the fixed sampling route in MA/PL_V2 by random sampling route. Obviously, the comparisons of these variants and MA/PL can reveal the effectiveness of the learning rule for JPD, the random sampling route and LS_LUF. For fair comparison, we first run MA/PL with genMax $=5000$ and record the CPU time. Then, the other variants are run for the same CPU time. Note that parameters in the variants are the same as in MA/PL. Comparison results are listed in Table I. In terms of the percentage deviations from BKS, i.e., $\operatorname{Dev}(\%)$, the related violin plots are given in Fig. 7.

TABLE I

COMPARISONS OF MA/PL V1, MA/PL V2, MA/PL V3 AND MA/PL

\begin{tabular}{|c|c|c|c|c|c|c|c|c|}
\hline \multirow{2}{*}{ Instance } & \multicolumn{2}{|c|}{ MA/PL V1 } & \multicolumn{2}{|c|}{ MA/PL V2 } & \multicolumn{2}{|c|}{ MA/PL V3 } & \multicolumn{2}{|l|}{ MA/PL } \\
\hline & $A V G$ & $S D$ & $A V G$ & $S D$ & $A V G$ & $S D$ & $A V G$ & $S D$ \\
\hline$p 1$ & 3417.1 & 9 & & 2.51 & & & .0 & 0. \\
\hline p2 & & 7. & & 4.49 & & & & 00 \\
\hline 1 & .1 & 4.76 & .9 & 5.92 & 3090.0 & 0. & 0.0 & 0.00 \\
\hline 2 & 2 & 6.39 & .2 & 6.39 & .0 & 5. & 186.0 & 0.00 \\
\hline 4 & 94799.0 & 6.00 & 95168.0 & 0.00 & 95168.0 & .0 & 168.0 & 0.00 \\
\hline & 378 & 6.64 & & 0.00 & & & .0 & 0.00 \\
\hline & & 6 & & 4.20 & & & & .00 \\
\hline & & 0 & & 2.94 & & 0.50 & & 0.0 \\
\hline t2 & .0 & 0.00 & 1.0 & 0.00 & 1.0 & 0.00 & 1.0 & 0.00 \\
\hline t3 & 4 & 0.00 & 40 & 0.00 & 40 & 0. & .0 & 0.00 \\
\hline & 6 & 0.00 & 6 & 0.00 & 612 & 0.00 & 0.0 & 0.00 \\
\hline & & .00 & & 2.49 & & & 0.0 & 0.00 \\
\hline & & & & 2.51 & & & & 0.00 \\
\hline & & 1 & & 18.40 & & & & 0.0 \\
\hline & 23003.0 & 39. & 230 & 30.2 & 23 & 14 & 46.8 & 15. \\
\hline & 9 & 37. & 227 & 51.7 & 722 & 27.47 & 50.7 & 28.17 \\
\hline & 7.1 & 66. & 220 & 46.9 & 22 & 47 & 22105.1 & 28.1 \\
\hline & 22602.1 & 49.8 & 22596.1 & 39. & 522 & 50. & 22714.0 & 12.4 \\
\hline & & 103 & & 96 & & & 6.7 & 39 \\
\hline & & 29. & & 54. & & & & 43.9 \\
\hline & & 20. & & 5.2 & & & & \\
\hline & & 83. & & 49.23 & & & 6.7 & 39. \\
\hline & & 27 & 222 & 44. & 22 & 38 & 8.1 & 15. \\
\hline & 22547.2 & 30 & 22547.2 & 30. & 22555.3 & 26 & 22558.2 & 14.9 \\
\hline Best & 3 & 4 & & 6 & 9 & 10 & 24 & 21 \\
\hline & & & & & & & & \\
\hline
\end{tabular}

Note: The $p$-values of "MA/PL_V2 vs. MA/PL_V1" and "MA/PL_V3 vs. MA/PL_V2" are 0.012 and 0.000 , respectively. 
From Table I, we can see that MA/PL_V2 outperforms MA/PL_V1, clearly showing that the integration of MPD and JPD enhances the search ability. That is, considering the dependency of conjoint items leads to better the global exploration. Meanwhile, MA/PL V3 outperforms MA/PL V2 for almost all the instances in terms of $A V G$. Especially, there are a total of 13 instances for which the $S D$ values of MA/PL_V3 are smaller than those of MA/PL_V2. Thus, by capturing the potential dependencies among all items, the random sampling route improves the effectiveness and the robustness of MA/PL. Moreover, MA/PL outperforms MA/PL_V3 which demonstrates the effectiveness of the proposed LS_LLUF. From Fig. 7, it is clear that the distributions of MA/PL_V3 are better than MA/PL_V1 and MA/PL are better than MA/PL_V3, in terms of both the dispersions and the interquartile ranges.

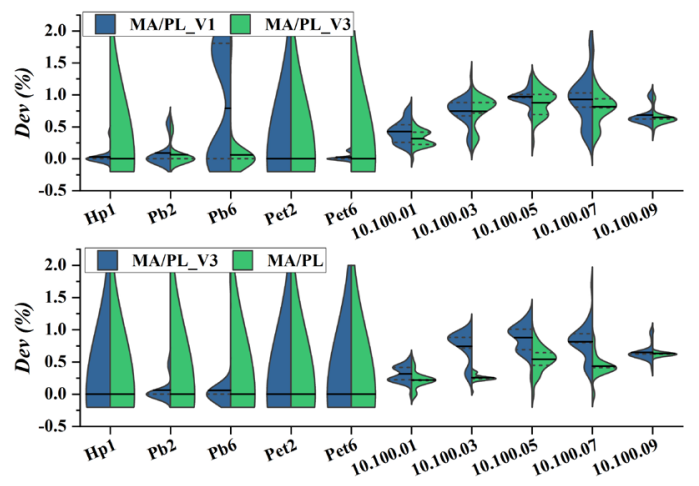

Fig. 7. Violin plots for considered comparisons

In addition, we also observe the $95 \% \mathrm{CI}$ for the solutions of MA/PL and the three variants for the other instances, i.e., Hp2, Knap 28, MK_gk04, Pb6, Pet7 and Sento2. The related CI's are shown in Fig. 8. We can see that MA/PL can obtain relatively more consistent solutions than the other three variants. These CI's also reveal the performance of MA/PL's components.

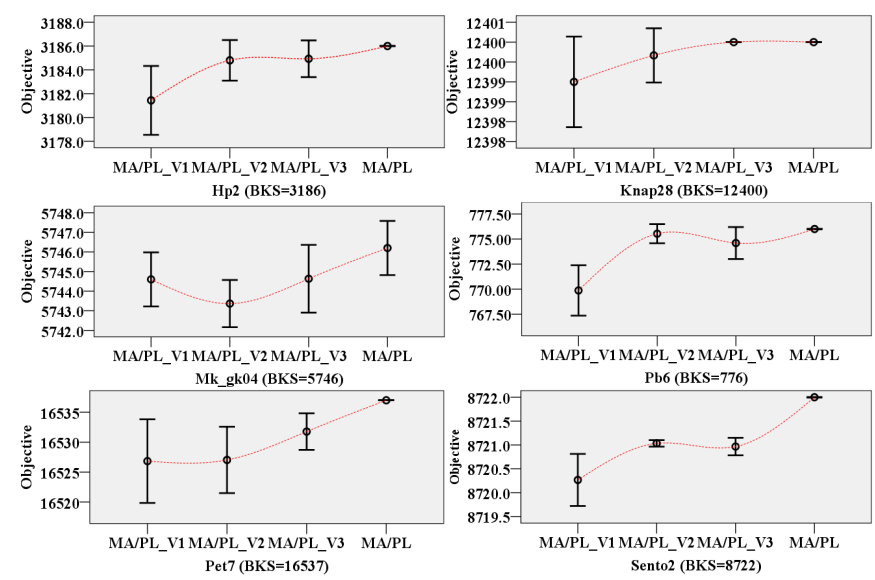

Fig. 8. Error bars $(95 \% \mathrm{CI})$ for MA/PL (V1 V3) and MA/PL

To further reveal the effectiveness of the proposed LS_LUF, two variants of MA/PL that use advanced local search methods in existing works for MKP are introduced. The two variants are MA/PL_NLS and MA/PL_ILS, which are the same as MA/PL except that LS_LUF is replaced by the neighborhood local search [62] and the intensity-based local search [34]. The same CPU times used in Table I are adopted for each algorithm. Results are given in Table II. In addition, in Fig. 9, we give the $95 \% \mathrm{CI}$ for the solutions of MA/PL and the two variants for the same instances that are shown in Fig. 8. The results of Table II and Fig. 9 reveal the competitiveness of the proposed LS_LUF.

2) Effectiveness of proposed repair operator

To verify the effectiveness and efficiency of the proposed RO_LUF, we compare MA/PL with two variants, namely, MA/PL_RO2 and MA/PL_RO3. They are the same as MA/PL except that RO_LUF is replaced by RO2 [34] and RO3 [35]. $\mathrm{RO} 2$ and $\mathrm{RO} 3$ are all the most effective repair heuristics for MKP in existing works. Note that the same stopping criterion (genMax $=5000$ ) is used for two variants and MA/PL. Relevant results on Test set II are reported in Table III. Meanwhile, the average CPU times for three algorithms are given in Fig. 10.

TABLE II

COMPARISONS OF MA/PL_NLS, MA/PL_ILS AND MA/PL

\begin{tabular}{|c|c|c|c|c|c|c|}
\hline \multirow{2}{*}{ Instance } & \multicolumn{2}{|c|}{ MA/PL NLS } & \multicolumn{2}{|c|}{ MA/PL ILS } & \multicolumn{2}{|l|}{$\mathrm{MA} / \mathrm{PL}$} \\
\hline & $A V G$ & $S D$ & $A V G$ & $S D$ & $A V G$ & $S D$ \\
\hline$\overline{\mathrm{Hp} 1}$ & 3416.7 & 3.83 & 3416.9 & 3.22 & 3418.0 & 0.00 \\
\hline $\mathrm{Hp} 2$ & 3180.5 & 9.88 & 3183.5 & 5.91 & 3186.0 & 0.00 \\
\hline $\mathrm{Pb} 1$ & 3090.0 & 0.00 & 3089.9 & 0.97 & 3090.0 & 0.00 \\
\hline $\mathrm{Pb} 2$ & 3182.6 & 6.56 & 3183.2 & 6.06 & 3186.0 & 0.00 \\
\hline $\mathrm{Pb} 4$ & 95167.9 & 0.51 & 94797.5 & 9.62 & 95168.0 & 0.00 \\
\hline $\mathrm{Pb} 5$ & 2138.9 & 0.37 & 2138.8 & 0.91 & 2139.0 & 0.00 \\
\hline $\mathrm{Pb} 6$ & 772.7 & 6.02 & 772.8 & 5.86 & 776.0 & 0.00 \\
\hline $\mathrm{Pb} 7$ & 1034.6 & 0.50 & 1033.9 & 2.22 & 1035.0 & 0.00 \\
\hline Pet2 & 87061.0 & 0.00 & 87061.0 & 0.00 & 87061.0 & 0.00 \\
\hline Pet3 & 4015.0 & 0.00 & 4015.0 & 0.00 & 4015.0 & 0.00 \\
\hline Pet4 & 6120.0 & 0.00 & 6120.0 & 0.00 & 6120.0 & 0.00 \\
\hline Pet5 & 12398.9 & 3.09 & 12399.3 & 1.86 & 12400.0 & 0.00 \\
\hline Pet6 & 10616.5 & 4.17 & 10616.6 & 3.97 & 10618.0 & 0.00 \\
\hline Pet7 & 16533.7 & 6.89 & 16532.8 & 7.75 & 16537.0 & 0.00 \\
\hline 10.100 .00 & 23028.9 & 30.47 & 23025.9 & 32.62 & 23046.8 & 15.82 \\
\hline 10.100 .01 & 22724.8 & 34.73 & 22722.9 & 51.61 & 22750.7 & 28.17 \\
\hline 10.100 .02 & 22049.8 & 77.84 & 22061.2 & 62.58 & 22105.1 & 28.10 \\
\hline 10.100 .03 & 22691.1 & 53.64 & 22700.1 & 45.02 & 22714.0 & 12.44 \\
\hline 10.100 .04 & 22708.7 & 82.10 & 22701.2 & 67.14 & 22726.7 & 39.59 \\
\hline 10.100 .05 & 22611.9 & 77.62 & 22606.2 & 55.79 & 22653.9 & 43.92 \\
\hline 10.100 .06 & 21747.3 & 75.01 & 21753.7 & 73.76 & 21804.0 & 54.43 \\
\hline 10.100 .07 & 22483.8 & 100.09 & 22502.2 & 70.28 & 22536.7 & 39.08 \\
\hline 10.100 .08 & 22422.0 & 112.19 & 22425.3 & 117.37 & 22508.1 & 15.80 \\
\hline 10.100 .09 & 22530.6 & 40.68 & 22533.3 & 39.80 & 22558.2 & 14.90 \\
\hline \#Best & 4 & 4 & 3 & 3 & 24 & 24 \\
\hline$p$-value & 0.000 & & 0.000 & & & \\
\hline
\end{tabular}

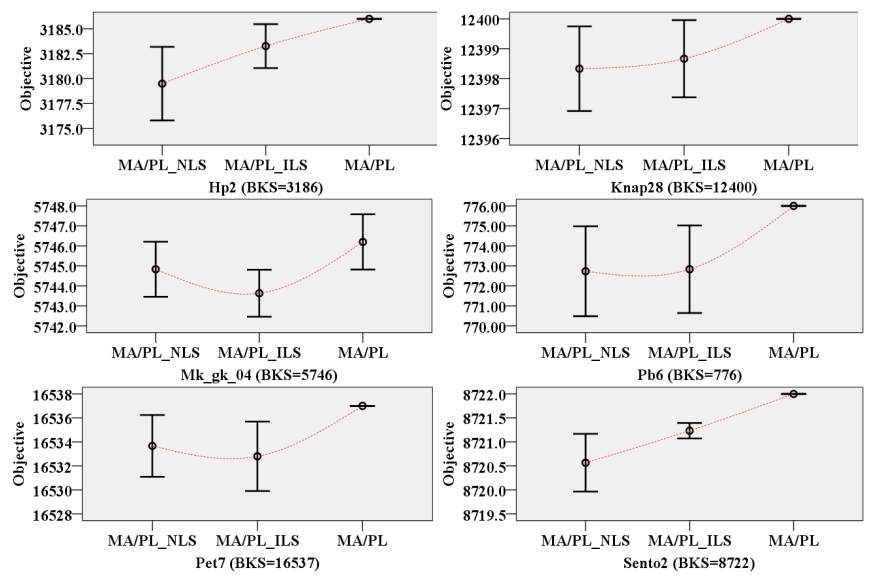

Fig. 9. Error bars $(95 \% \mathrm{CI})$ for MA/PL_NLS (ILS) and MA/PL 
TABLE III

COMPARISONS OF MA/PL RO2/3 AND MA/PL

\begin{tabular}{|c|c|c|c|c|c|c|c|}
\hline \multirow{2}{*}{ Instance } & \multirow{2}{*}{ BKS } & \multicolumn{2}{|c|}{ MA/PL RO2 } & \multicolumn{2}{|c|}{ MA/PL RO3 } & \multicolumn{2}{|l|}{$\mathrm{MA} / \mathrm{PL}$} \\
\hline & & BEST & $A V G$ & BEST & $A V G$ & BEST & $A V G$ \\
\hline 5.100 & 381 & 24381 & 24336.97 & 4381 & 24380.20 & 27501 & 2438 \\
\hline & 24274 & 274 & 50 & 4274 & 242 & $24274^{*}$ & 427 \\
\hline & 23551 & & 2352 & & 235 & & 23519.97 \\
\hline & & & & & & & \\
\hline & & & & & & & 43 \\
\hline & 2 & 4601 & 53 & 8 & 24 & & 90 \\
\hline & & & 23 & & & & 255 \\
\hline & 23410 & & & & & & \\
\hline & 24216 & & & & & & \\
\hline & & & & & & & \\
\hline & & & & & & & \\
\hline & 1 & 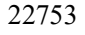 & & 6 & 22 & & 22 \\
\hline & 131 & 131 & 22 & 22 & 22 & 22 & 22 \\
\hline & 772 & 2718 & 7 & 22763 & 226 & 22 & 227 \\
\hline & 75 & 22654 & 226 & 22751 & 226 & $22751^{*}$ & 22726.73 \\
\hline & 2777 & 22702 & 226 & 22777 & 226 & $22777^{*}$ & 22653.90 \\
\hline & 875 & 21786 & & 21791 & & & 21803.97 \\
\hline & 635 & 2 & & 22 & & & 225 \\
\hline & & 3 & & & & & \\
\hline & 702 & & & 22559 & 225 & & 225 \\
\hline & & 8 & 2 & 10 & & 19 & 16 \\
\hline & & 0.002 & 0.007 & 0.002 & & & \\
\hline
\end{tabular}

Table III shows that MA/PL is better than MA/PL_RO2/3 for almost all the instances, regarding BEST and $A V G$. Hence, RO_LUF is beneficial to enhance the overall solution quality of MA/PL. Besides, as shown in Fig. 10, MA/PL takes less CPU time than MA/PL_RO2/3 although the same stopping criterion is adopted. These results prove the effectiveness and efficiency of the proposed RO_LUF. In terms of Dev (\%), the violin plots of MA/PL and MA/PL_RO2/3 are given in Fig. 11, showing that MA/PL can obtain more robust solutions than the variants.

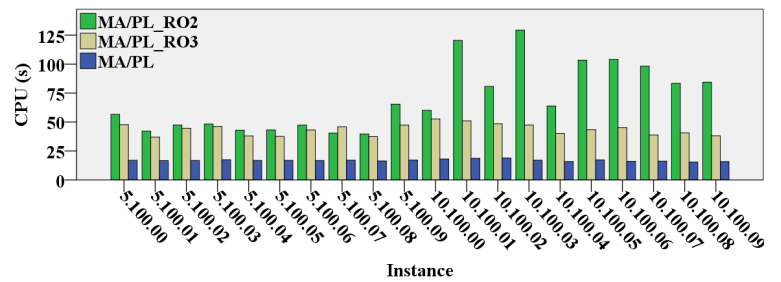

Fig. 10. Average CPU times for MA/PL_RO2/3 and MA/PL

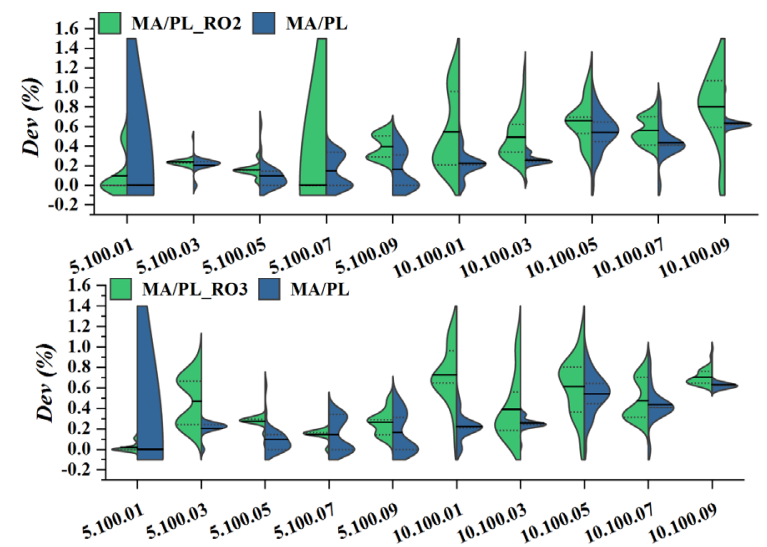

Fig. 11. Violin plots for solutions of MA/PL_RO2/3 and MA/PL

\section{3) Discussions on coefficients for RO_LUF and LS_LUF}

The coefficients $\lambda_{1}$ and $\lambda_{2}$ applied for calculating LUFs play an important role on the performance of RO LUF and LS_LUF. To study the impacts of $\lambda_{1}$ and $\lambda_{2}$, we introduce four variants of MA/PL, i.e., MA/PL_A, MA/PL_B, MA/PL_C and MA/PL_D, with respect to different combinations of $\left(\bar{\lambda}_{1}, \lambda_{2}\right)$. The values of $\left(\lambda_{1}, \lambda_{2}\right)$ for these four variants are $(0.1,0.9)$, $(0.3,0.7),(0.7,0.3)$ and $(0.9,0.1)$. We then assess the effectiveness and the efficiency of MA/PL and the four variants. The comparative results in terms of $A V G$ are reported in Table IV. In addition, we give trends of $S D$ in Fig. 12. Note that the four variants are executed with genMax $=5000$ which is the same as MA/PL.

TABLE IV COMPARISONS OF FOUR MA/PL (A D) AND MA/PL

\begin{tabular}{llllll}
\hline \hline \multirow{2}{*}{ Instance } & MA/PL_A & MA/PL_B & MA/PL_C & MA/PL__ & MA/PL \\
\cline { 2 - 6 } & $A V G$ & $A V G$ & $A V G$ & $A V G$ & $A V G$ \\
\hline 5.100 .00 & 24338.8 & 24341.8 & 24348.8 & 24341.6 & $\mathbf{2 4 3 8 0 . 6 7}$ \\
5.100 .01 & 24236.0 & 24233.9 & 24243.2 & 24238.8 & $\mathbf{2 4 2 7 4 . 0 0}$ \\
5.100 .02 & 23508.2 & 23509.1 & 23508.2 & 23509.1 & $\mathbf{2 3 5 1 9 . 9 7}$ \\
5.100 .03 & 23440.3 & 23439.7 & 23460.5 & 23454.7 & $\mathbf{2 3 4 8 6 . 0 0}$ \\
5.100 .04 & 23915.2 & 23924.5 & 23941.3 & 23937.2 & $\mathbf{2 3 9 6 2 . 4 3}$ \\
5.100 .05 & 24574.9 & 24573.5 & 24574.5 & 24572.6 & $\mathbf{2 4 5 8 8 . 9 0}$ \\
5.100 .06 & 25503.1 & 25475.4 & 25517.3 & 25505.3 & $\mathbf{2 5 5 5 8 . 3 3}$ \\
5.100 .07 & 23353.6 & 23349.4 & 23350.4 & 23348.7 & $\mathbf{2 3 3 7 6 . 1 7}$ \\
5.100 .08 & 24165.1 & 24196.3 & 24206.0 & 24206.4 & $\mathbf{2 4 2 0 8 . 4 0}$ \\
5.100 .09 & 24325.4 & 24331.0 & 24333.3 & 24335.5 & $\mathbf{2 4 3 7 1 . 3 7}$ \\
10.100 .00 & 23026.8 & 23029.0 & 23036.9 & 23036.9 & $\mathbf{2 3 0 4 6 . 8 3}$ \\
10.100 .01 & 22723.4 & 22714.6 & 22735.9 & 22736.2 & $\mathbf{2 2 7 5 0 . 7 3}$ \\
10.100 .02 & 22075.0 & 22067.9 & 22085.3 & 22080.8 & $\mathbf{2 2 1 0 5 . 1 3}$ \\
10.100 .03 & 22664.2 & 22660.3 & 22662.7 & 22666.2 & $\mathbf{2 2 7 1 4 . 0 3}$ \\
10.100 .04 & 22659.4 & 22670.9 & 22652.3 & 22669.7 & $\mathbf{2 2 7 2 6 . 7 3}$ \\
10.100 .05 & 22620.8 & 22630.3 & 22630.3 & 22627.1 & $\mathbf{2 2 6 5 3 . 9 0}$ \\
10.100 .06 & 21767.8 & 21766.1 & 21768.1 & 21768.1 & $\mathbf{2 1 8 0 3 . 9 7}$ \\
10.100 .07 & 22501.8 & 22502.8 & 22515.4 & 22506.9 & $\mathbf{2 2 5 3 6 . 6 7}$ \\
10.100 .08 & 22450.0 & 22453.5 & 22446.9 & 22451.1 & $\mathbf{2 2 5 0 8 . 0 7}$ \\
10.100 .09 & 22558.2 & 22552.7 & 22556.8 & $\mathbf{2 2 5 6 0 . 5}$ & 22558.23 \\
\#Best & 0 & 0 & 0 & 1 & $\mathbf{1 9}$ \\
$p$-value & 0.000 & 0.000 & 0.000 & 0.000 & \\
\hline \hline
\end{tabular}

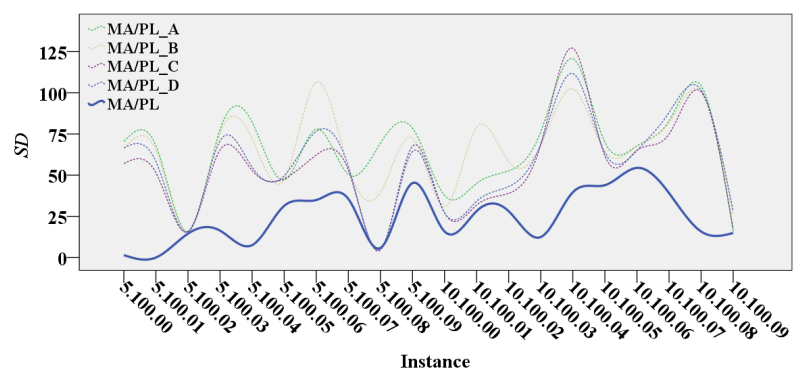

Fig. 12. Trends of $S D$ for MA/PL (A D ) and MA/PL

Table IV shows that the values of $A V G$ of MA/PL are much better than those of the four variants for almost all the used instances. The results verify that in RO_LUF and LS_LUF the equal weight of the two attributes for candidate items is helpful in enhancing the effectiveness of MA/PL. Meanwhile, in Fig. 12, MA/PL has relatively smaller $S D$ for each instance, so setting the equal coefficient to the two attributes is beneficial to enhance the robustness of MA/PL as well. This can be due to the fact that for the proposed LUFs the different magnitude of the two attributes has already been normalized before using them. Thereby, the equal coefficient of them is more reliable to reflect the problem-specific relationships of the two attributes.

Moreover, we draw the trend of CPU (s) for each algorithm 
in Fig. 13. It is clear that algorithms with larger $\lambda_{2}$ tend to take less CPU time. This is because, during the search process of MA/PL, a larger $\lambda_{2}$ that corresponds to a smaller $\lambda_{1}$ will emphasize the role of the constraint violation but weaken the impact of the objective. Thereby, for MA/PL with a larger $\lambda_{2}$, the time complexities of RO LUF and LS LUF can be much lower than the worst case $O(P S \cdot N \cdot(3 \cdot N+M))$, compared with ones using smaller $\lambda_{2}$. Especially, the trend line of MA/PL in Fig. 13 is located in the relatively middle area among all the lines. However, the CPU time for MA/PL is quite acceptable as we can see in this section. Therefore, we set $\left(\lambda_{1}, \lambda_{2}\right)=$ $(0.5,0.5)$ considering both the effectiveness and efficiency of $\mathrm{MA} / \mathrm{PL}$.

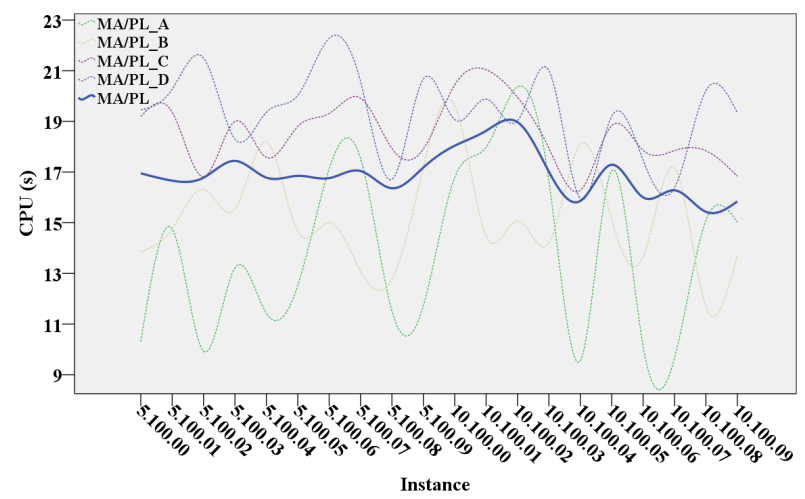

Fig. 13. Trends of CPU time for MA/PL (A D) and MA/PL

\section{Comparisons with Learning-based Metaheuristics}

We compare MA/PL with four learning-based metaheuristics for MKP, i.e., HEDA1 [34], HEDA2 [34], Meta-RaPS EDA [55] and Meta-RaPS Q [55]. HEDA1 and HEDA2 use the EDA-based learning scheme. Whereas HEDA2 uses RO2 as the repair operator, HEDA1 uses a repair operator based on LP. Meta-RaPS EDA and Meta-RaPS Q adopt a model of memoryless metaheuristic (i.e., Meta-RaPS). Meta-RaPS EDA and Meta-RaPS Q employ EDA and Q-learning as the underlying algorithms. These algorithms are the most currently reported learning-based ones for MKP. The stopping criterion of MAPL is genMax $=5000$. Relevant results are listed in Tables V to VI.

TABLE V

COMPARISONS OF HEDA1, HEDA2 AND MA/PL

\begin{tabular}{llllllll}
\hline \multicolumn{7}{c}{ Instance } & \multicolumn{7}{c}{ HEDA1 } & \multicolumn{5}{c}{ HEDA2 } & \multicolumn{7}{c}{ MA/PL } \\
\cline { 2 - 8 } Ave. Dev & Var. Dev Ave. Dev & Var. Dev Ave. Dev & Var. Dev CPU (s) \\
\hline Mk_gk01 & 0.32 & 0.08 & 0.94 & 0.66 & $\mathbf{0 . 2 3}$ & $\mathbf{0 . 0 0}$ & 20.07 \\
Mk_gk02 & $\mathbf{0 . 2 0}$ & 0.12 & 0.98 & 0.40 & 0.25 & $\mathbf{0 . 0 0}$ & 22.77 \\
Mk_gk03 & $\mathbf{0 . 2 0}$ & 0.10 & 1.15 & 0.78 & 0.40 & $\mathbf{0 . 0 0}$ & 40.83 \\
Mk_gk04 & 0.34 & 0.29 & 1.27 & 0.71 & $\mathbf{0 . 3 1}$ & $\mathbf{0 . 0 0}$ & 52.73 \\
Mk_gk05 & 0.28 & 0.22 & 1.50 & 0.75 & $\mathbf{0 . 2 3}$ & $\mathbf{0 . 0 0}$ & 57.19 \\
Mk_gk06 & 0.44 & 0.23 & 1.44 & 1.16 & $\mathbf{0 . 3 5}$ & $\mathbf{0 . 0 0}$ & 78.95 \\
Mk_gk07 & 0.72 & 0.82 & 1.85 & 0.99 & $\mathbf{0 . 2 8}$ & $\mathbf{0 . 0 0}$ & 211.11 \\
Mk_gk08 & 0.77 & 1.10 & 1.73 & 0.29 & $\mathbf{0 . 4 0}$ & $\mathbf{0 . 0 0}$ & 348.42 \\
Mk_gk09 & - & - & 2.55 & 0.68 & $\mathbf{0 . 2 4}$ & $\mathbf{0 . 0 0}$ & 1204.68 \\
Mk_gk10 & - & - & 2.12 & 0.78 & $\mathbf{0 . 8 1}$ & $\mathbf{0 . 0 0}$ & 1779.39 \\
Mk_gk11 & - & - & 1.74 & 0.22 & $\mathbf{0 . 0 1}$ & $\mathbf{0 . 0 0}$ & 2411.12 \\
Knap15 & - & - & $\mathbf{0 . 0 0}$ & $\mathbf{0 . 0 0}$ & $\mathbf{0 . 0 0}$ & $\mathbf{0 . 0 0}$ & 2.02 \\
Knap20 & - & - & $\mathbf{0 . 0 0}$ & $\mathbf{0 . 0 0}$ & $\mathbf{0 . 0 0}$ & $\mathbf{0 . 0 0}$ & 2.72 \\
Knap39 & - & - & $\mathbf{0 . 0 0}$ & $\mathbf{0 . 0 0}$ & 0.03 & $\mathbf{0 . 0 0}$ & 5.42 \\
Knap28 & - & - & $\mathbf{0 . 0 0}$ & $\mathbf{0 . 0 0}$ & $\mathbf{0 . 0 0}$ & $\mathbf{0 . 0 0}$ & 3.76 \\
\#Best & 2 & 0 & 4 & 4 & $\mathbf{1 2}$ & $\mathbf{1 5}$ & \\
p-value & 0.157 & & 0.004 & & & & \\
\hline \hline
\end{tabular}

TABLE VI

COMPARISONS OF META-RAPS EDA, META-RAPS Q AND MA/PL

\begin{tabular}{lllll}
\hline \hline \multirow{2}{*}{ Instance } & RaPS-EDA & RaPS-Q & MA/PL & \\
\cline { 2 - 5 } & Ave. Dev & Ave. Dev & Ave. Dev & CPU (s) \\
\hline Hp1 & $\mathbf{0 . 0 0}$ & 0.09 & $\mathbf{0 . 0 0}$ & 3.73 \\
Hp2 & $\mathbf{0 . 0 0}$ & 0.19 & $\mathbf{0 . 0 0}$ & 4.68 \\
Pb1 & $\mathbf{0 . 0 0}$ & 0.09 & $\mathbf{0 . 0 0}$ & 3.68 \\
Pb2 & $\mathbf{0 . 0 0}$ & 0.52 & $\mathbf{0 . 0 0}$ & 4.47 \\
Pet6 & 0.04 & 0.20 & $\mathbf{0 . 0 2}$ & 6.04 \\
Pet7 & $\mathbf{0 . 0 0}$ & 0.19 & $\mathbf{0 . 0 0}$ & 7.98 \\
Sento2 & $\mathbf{0 . 0 0}$ & 0.02 & $\mathbf{0 . 0 0}$ & 10.64 \\
Weing6 & $\mathbf{0 . 0 0}$ & 0.06 & $\mathbf{0 . 0 0}$ & 4.13 \\
Weing7 & $\mathbf{0 . 0 0}$ & 0.05 & $\mathbf{0 . 0 0}$ & 16.30 \\
Weing8 & 0.05 & 0.31 & $\mathbf{0 . 0 0}$ & 18.12 \\
Weish18 & $\mathbf{0 . 0 0}$ & 0.13 & $\mathbf{0 . 0 0}$ & 10.86 \\
Weish22 & $\mathbf{0 . 0 0}$ & 0.07 & $\mathbf{0 . 0 0}$ & 12.72 \\
Weish23 & $\mathbf{0 . 0 0}$ & 0.02 & $\mathbf{0 . 0 0}$ & 12.83 \\
Weish24 & $\mathbf{0 . 0 0}$ & 0.20 & $\mathbf{0 . 0 0}$ & 12.61 \\
Weish25 & $\mathbf{0 . 0 0}$ & 0.09 & $\mathbf{0 . 0 0}$ & 12.67 \\
Weish26 & $\mathbf{0 . 0 0}$ & 0.09 & $\mathbf{0 . 0 0}$ & 14.39 \\
\#Best & 14 & 0 & 16 & \\
p-value & 0.157 & 0.000 & & \\
\hline \hline
\end{tabular}

From Table V, we can see that MA/PL outperforms HEDA1 and HEDA2. Although MA/PL's learning mechanism is similar to HEDA1 and HEDA2, its probability distribution is estimated based on ExA, rather than the elite individuals in the population. Such a learning method of MA/PL can make good use of the information of elite individuals and so speeds up the convergence. Besides, the integration of MPD and JPD can capture valuable probability information to guide the global exploration of MA/PL. We can see from Table VI that MA/PL outperforms Meta-RaPS EDA and Meta-RaPS Q. For all the instances where any of other methods found BKS, MA/PL also found BKS, while for all other instances MA/PL found better solutions. Thus, our learning method is effective for MKP. It may be worthwhile to consider embedding our learning method into other metaheuristics for MKP in future research.

\section{Comparisons with Other Existing Algorithms}

In this subsection, we compare MA/PL with 9 existing algorithms for MKP with respect to Test sets I to IV. These existing algorithms are listed in Table VII, which are all the most currently effective solution methods for MKP. The stopping criterion of MA/PL is genMax $=5000$.

TABLE VII

EXISTING ALGORITHMS CONSIDERED IN THE COMPARISONS

\begin{tabular}{|c|c|c|c|c|c|}
\hline \multirow{2}{*}{ Algorithm } & \multirow{2}{*}{ Published year } & \multicolumn{4}{|c|}{ Test set } \\
\hline & & $\overline{\mathrm{I}}$ & II & III & IV \\
\hline NBHS2 [53] & 2015 & $\sqrt{ }$ & $x$ & $x$ & $x$ \\
\hline TR-BDS [52] & 2016 & $\sqrt{ }$ & $\times$ & $\sqrt{ }$ & $x$ \\
\hline QIFAPSO [49] & 2016 & $x$ & $\sqrt{ }$ & $x$ & $x$ \\
\hline $\mathrm{BCSA}[51]$ & 2017 & $\sqrt{ }$ & $x$ & $\sqrt{ }$ & $x$ \\
\hline HPSOGO [50] & 2018 & $x$ & $x$ & $\sqrt{ }$ & $\times$ \\
\hline KMTR-Cuckoo [54] & 2018 & $x$ & $\times$ & $x$ & $\sqrt{ }$ \\
\hline KMTR-BH [54] & 2018 & $x$ & $x$ & $x$ & $\sqrt{ }$ \\
\hline HBDE-RO3 [35] & 2019 & $x$ & $\sqrt{ }$ & $\sqrt{ }$ & $x$ \\
\hline MMVO [60] & 2019 & $\times$ & $\sqrt{ }$ & $\times$ & $\times$ \\
\hline
\end{tabular}

Note: " ل" (“×") means the test set is relevant (irrelevant) to the algorithm.

\section{1) Computational results for Test set I}

The comparative results on Test set I are reported in Table VIII. We can see from Table VIII that MA/PL outperforms TR-BDS, regarding $A V G$ and $S D$, for almost all the instances of 
Test set I. Although the Min. Dev and Ave. Dev values of MA/PL are similar to those of NBHS2, MA/PL's Var. Dev values are all less than 0.01 and are all smaller than those of NBHS2. Thus, MA/PL can maintain better robustness than NBHS2. There are three instances, i.e., MK_gk03, MK_gk04 and MK_gk05 for which BCSA can provide smaller Ave. Dev. However, BCSA is somewhat weak in other instances and may be unavailable for large scale instances MK_gk10 and MK_gk_11. Moreover, the CPU times for MA/PL are all less than 1205s from MK_gk01 to MK_gk09 which are quite acceptable. Furthermore, for large-sized instances MK_gk10 and MK_gk_11, MA/PL is able to obtain high-quality solutions with smaller $A V G, S D$ and Var. Dev. In view of the above analysis, the proposed MA/PL can be potentially used for large-sized instances of MKP.

\section{2) Computational results for Test set II}

In terms of Test set II, comparative results are listed in Table IX. It is clear that MA/PL achieves a good performance for almost all the considered instances in terms of $B E S T, A V G$ and $S D$. For the significance, the $p$-values for three comparative algorithms are all smaller than 0.05 concerned with BEST and $A V G$, except for the $A V G$ values of QIFAPSO. However, MA/PL can attain the BKS values for 15 of 20 instances which is much better than QIFAPSO. Moreover, the CPU (s) values listed in Tables IX range from $15 \mathrm{~s}$ to $19 \mathrm{~s}$, which verify the efficiency of the proposed MA/PL. Therefore, the proposed $\mathrm{MA} / \mathrm{PL}$ is effective for medium scale instances of MKP.

TABLE VIII

COMPARISONS OF BHS2, BCSA, TR-BDS AND MA/PL (VS. TEST SET I)

\begin{tabular}{|c|c|c|c|c|c|c|c|c|c|c|c|c|c|c|}
\hline \multirow{2}{*}{ Instance } & \multirow{2}{*}{ BKS } & \multicolumn{3}{|l|}{ NBHS2 } & \multicolumn{2}{|l|}{ BCSA } & \multicolumn{2}{|l|}{ TR-BDS } & \multicolumn{6}{|l|}{$\overline{\mathrm{MA} / \mathrm{PL}}$} \\
\hline & & Min. Dev & Ave. Dev & Var. Dev & Ave. Dev & Var. Dev & $A V G$ & $S D$ & $A V G$ & $S D$ & Min. & Dev Ave. Dev & Var. Dev & $\mathrm{CPU}(\mathrm{s})$ \\
\hline Mk_gk02 & 3958 & 0.18 & 0.30 & 0.08 & 0.27 & 0.08 & 3878.88 & 8.09 & 3948.00 & 2.75 & 0.08 & 0.25 & $<0.01$ & 22.77 \\
\hline Mk_gk05 & 7557 & 0.16 & 0.39 & 0.11 & 0.17 & 0.08 & 7349.14 & 11.41 & 7539.70 & 4.69 & 0.11 & 0.23 & $<0.01$ & 57.19 \\
\hline Mk_gk06 & 7672 & 0.20 & 0.42 & 0.17 & 0.35 & 0.12 & 7488.88 & 9.95 & 7645.10 & 4.23 & 0.25 & 0.35 & $<0.01$ & 78.95 \\
\hline $\mathrm{Mk}$ gk07 & 19215 & 0.15 & 0.23 & 0.06 & 0.70 & 0.12 & 18588.20 & 22.74 & 19161.27 & 6.02 & 0.23 & 0.28 & $<0.01$ & 211.11 \\
\hline Mk_gk10 & 57292 & 0.38 & 0.53 & 0.06 & - & - & 55719.48 & 25.50 & 56825.43 & 23.07 & 0.33 & 0.81 & $<0.01$ & 1779.39 \\
\hline Mk_gk11 & 95231 & 0.46 & 0.64 & 0.09 & - & - & 93132.92 & 35.92 & 94124.03 & 0.94 & 0.01 & 0.01 & $<0.01$ & 2411.12 \\
\hline \#Best & & 6 & 2 & 0 & 5 & 0 & 1 & 0 & 10 & 11 & 6 & 6 & 11 & \\
\hline$p$-value & & 1.000 & 0.035 & & 0.705 & & 0.001 & & & & & & & \\
\hline
\end{tabular}

TABLE IX

COMPARISONS OF HBDE-RO3, MMVO, QIFAPSO AND MA/PL (VS. TEST SET II)

\begin{tabular}{|c|c|c|c|c|c|c|c|c|c|c|c|c|}
\hline \multirow{2}{*}{ Instance } & \multirow{2}{*}{ BKS } & \multicolumn{3}{|c|}{ HBDE-RO3 } & \multicolumn{2}{|c|}{ MMVO } & \multicolumn{2}{|c|}{ "QIFAPSO } & \multicolumn{4}{|l|}{ 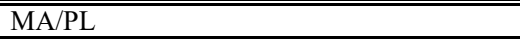 } \\
\hline & & $\overline{B E S T}$ & $A V G$ & $S D$ & $B E S T$ & $A V G$ & $B E S T$ & $A V G$ & $B E S T$ & $A V G$ & $S D$ & CPU (s) \\
\hline 5.100 .01 & 24274 & 24274 & 24250.4 & 61.01 & 24274 & 24274 & 24274 & 24206.0 & $24274^{*}$ & 24274.00 & 0.00 & 16.66 \\
\hline 5.100 .02 & 23551 & 23523 & 23490.7 & 33.44 & 23538 & 23520 & 23538 & 23513.0 & 23538 & 23519.97 & 14.50 & 16.78 \\
\hline 5.100 .04 & 23991 & 23966 & 23953.4 & 17.50 & 23947 & 23900 & 23991 & 23924.0 & 23991* & 23962.43 & 7.54 & 16.78 \\
\hline 5.100 .05 & 24613 & 24613 & 24597.9 & 8.97 & - & - & - & - & $24613^{*}$ & 24588.90 & 31.36 & 16.85 \\
\hline 5.100 .06 & 25591 & 25591 & 25522.4 & 9.80 & - & - & - & - & $25591^{*}$ & 25558.33 & 34.92 & 16.76 \\
\hline 10.100 .00 & 23064 & 23055 & 23053.9 & 7.56 & 22805 & 22700 & 23057 & 22954.0 & 23057 & 23046.83 & 15.82 & 18.04 \\
\hline 10.100 .01 & 22801 & 22801 & 22735.4 & 51.55 & 22630 & 22480 & 22750 & 22649.0 & $22801^{*}$ & 22750.73 & 28.17 & 18.63 \\
\hline 10.100 .02 & 22131 & 22131 & 22098.2 & 32.76 & 22131 & 21720 & 22131 & 21977.0 & $22131^{*}$ & 22105.13 & 28.10 & 18.97 \\
\hline 10.100 .03 & 22772 & 22749 & 22693.2 & 43.87 & 22347 & 22160 & 22772 & 22566.0 & 22763 & 22714.03 & 12.44 & 17.01 \\
\hline 10.100 .04 & 22751 & 22751 & 22748.4 & 17.92 & 22417 & 22290 & 22654 & 22555.0 & $22751^{*}$ & 22726.73 & 39.59 & 15.86 \\
\hline 10.100 .05 & 22777 & 22698 & 22593.6 & 45.86 & - & - & - & - & $22777^{*}$ & 22653.90 & 43.92 & 17.29 \\
\hline 10.100 .06 & 21875 & 21856 & 21734.5 & 63.11 & - & - & - & - & 21856 & 21803.97 & 54.43 & 16.00 \\
\hline$p$-value & & 0.014 & 0.025 & & 0.008 & 0.020 & 0.317 & 0.002 & & & & \\
\hline
\end{tabular}

3) Computational results for Test set III

We report the results regarding Test set III in Table X, which only contains the most currently proposed algorithms, i.e., HBDE-RO3 and HPSOGO. Meanwhile, the \#Best of BEST, $A V G$ and $S D$ of TR-BDS, BCSA and MA/PL are given in Table $\mathrm{XI}$. It is worth noting that Table X MA/PL can get the BKS values for all instances of Test set III. In addition, the $S D$ values of MA/PL are all obviously smaller than those of HBDE-RO3 and HPSOGO. Table XI shows that MA/PL can obtain more competitive performance than TR-BDS and BCSA. The above facts prove the effectiveness and robustness of our MA/PL for solving the small scale instances of MKP. More details about the comparative results associated with Test set III are available at http://dx.doi.org/10.13140/RG.2.2.23779.71208. 
TABLE $X$

COMPARISONS OF HBDE-RO3, HPSOGO AND MA/PL (VS. TEST SET III)

\begin{tabular}{|c|c|c|c|c|c|c|c|c|c|}
\hline \multirow{2}{*}{ Instance } & \multirow{2}{*}{ BKS } & \multicolumn{4}{|c|}{ HBDE-RO3 HPSOGO } & \multicolumn{4}{|c|}{ MA/PL } \\
\hline & & $S R$ & $S D$ & $S R$ & Ave. $D$ & $S R$ & Ave. & $v S D$ & CPU (s) \\
\hline Sentol & 7772 & 1.00 & 0.00 & 0.25 & 0.00 & 1.00 & 0.00 & 0.00 & 10.34 \\
\hline Sento2 & 8722 & 0.91 & 0.29 & 0.10 & 0.00 & 1.00 & 0.00 & 0.00 & 10.64 \\
\hline Hp1 & 3418 & 1.00 & 0.00 & - & - & 1.00 & 0.00 & 0.00 & 3.73 \\
\hline $\mathrm{Hp} 2$ & 3186 & 1.00 & 0.00 & - & - & 1.00 & 0.00 & 0.00 & 4.68 \\
\hline $\mathrm{Pb} 1$ & 3090 & 1.00 & 0.00 & - & - & 1.00 & 0.00 & 0.00 & 3.68 \\
\hline $\mathrm{Pb} 2$ & 3186 & 1.00 & 0.00 & - & - & 1.00 & 0.00 & 0.00 & 4.47 \\
\hline $\mathrm{Pb} 4$ & 95168 & 1.00 & 0.00 & - & - & 1.00 & 0.00 & 0.00 & 3.74 \\
\hline $\mathrm{Pb} 5$ & 2139 & 1.00 & 0.00 & - & - & 1.00 & 0.00 & 0.00 & 2.71 \\
\hline $\mathrm{Pb} 6$ & 776 & 1.00 & 0.00 & - & - & 1.00 & 0.00 & 0.00 & 6.42 \\
\hline $\mathrm{Pb} 7$ & 1035 & 1.00 & 0.00 & - & - & 1.00 & 0.00 & 0.00 & 6.39 \\
\hline Pet2 & 87061 & - & - & - & - & 1.00 & 0.00 & 0.00 & 1.37 \\
\hline Pet3 & 4015 & - & - & - & - & 1.00 & 0.00 & 0.00 & 2.09 \\
\hline Pet4 & 6120 & - & - & - & - & 1.00 & 0.00 & 0.00 & 2.77 \\
\hline Pet5 & 12400 & - & - & - & - & $1.0 \mathrm{r} 0$ & 0.00 & 0.00 & 3.96 \\
\hline Pet6 & 10618 & - & - & - & - & 0.83 & 0.02 & 5.15 & 6.04 \\
\hline Pet7 & 16537 & - & - & - & - & 1.00 & 0.00 & 0.00 & 7.98 \\
\hline Weing1 & 141278 & 1.00 & 0.00 & 1.00 & 0.00 & 1.00 & 0.00 & 0.00 & 4.15 \\
\hline Weing2 & 130883 & 1.00 & 0.00 & 1.00 & 0.00 & 1.00 & 0.00 & 0.00 & 4.27 \\
\hline Weing3 & 95677 & 1.00 & 0.00 & 1.00 & 0.00 & 1.00 & 0.00 & 0.00 & 4.27 \\
\hline Weing4 & 119337 & 1.00 & 0.00 & 1.00 & 0.00 & 1.00 & 0.00 & 0.00 & 4.33 \\
\hline Weing5 & 98796 & 1.00 & 0.00 & 1.00 & 0.00 & 1.00 & 0.00 & 0.00 & 4.20 \\
\hline Weing6 & 130623 & 1.00 & 0.00 & 1.00 & 0.00 & 1.00 & 0.00 & 0.00 & 4.13 \\
\hline Weing7 & 1095445 & 1.00 & 0.00 & 1.00 & 0.11 & 0.23 & 0.00 & 0.00 & 16.30 \\
\hline Weing8 & 624319 & 1.00 & 0.00 & 1.00 & 1.34 & 1.00 & 0.00 & 0.00 & 18.12 \\
\hline Weish1 & 4554 & 1.00 & 0.00 & 1.00 & 0.00 & 1.00 & 0.00 & 0.00 & 4.67 \\
\hline Weish2 & 4536 & 1.00 & 0.00 & 1.00 & 0.00 & 1.00 & 0.00 & $\mathbf{0 . 0 0}$ & 4.65 \\
\hline Weish3 & 4115 & 1.00 & 0.00 & 1.00 & 0.00 & 1.00 & 0.00 & 0.00 & 4.70 \\
\hline Weish4 & 4561 & 1.00 & 0.00 & 1.00 & 0.00 & 1.00 & 0.00 & 0.00 & 4.62 \\
\hline Weish5 & 4514 & 1.00 & 0.00 & 1.00 & 0.00 & 1.00 & 0.00 & 0.00 & 4.76 \\
\hline Weish6 & 5557 & 0.91 & 3.72 & 1.00 & 0.00 & 1.00 & 0.00 & 0.00 & 6.45 \\
\hline Weish7 & 5567 & 1.00 & 0.00 & 1.00 & 0.00 & 1.00 & 0.00 & 0.00 & 6.41 \\
\hline Weish8 & 5605 & 1.00 & 0.00 & 1.00 & 0.00 & 1.00 & 0.00 & 0.00 & 6.46 \\
\hline Weish9 & 5246 & 1.00 & 0.00 & 1.00 & 0.00 & 1.00 & 0.00 & 0.00 & 6.43 \\
\hline Weish10 & 6339 & 1.00 & 0.00 & 1.00 & 0.00 & 1.00 & 0.00 & 0.00 & 8.26 \\
\hline Weish11 & 5643 & 1.00 & 0.00 & 1.00 & 0.00 & 1.00 & 0.00 & 0.00 & 7.82 \\
\hline Weish12 & 6339 & 1.00 & 0.00 & 0.35 & 0.00 & 1.00 & 0.00 & 0.00 & 7.73 \\
\hline Weish13 & 6159 & 1.00 & 0.00 & 1.00 & 0.00 & 1.00 & 0.00 & 0.00 & 7.80 \\
\hline Weish14 & 6954 & 1.00 & 0.00 & 1.00 & 0.00 & 1.00 & 0.00 & 0.00 & 9.38 \\
\hline Weish15 & 7486 & 1.00 & 0.00 & 1.00 & 0.00 & 1.00 & 0.00 & 0.00 & 9.41 \\
\hline Weish16 & 7289 & 1.00 & 0.00 & 1.00 & 0.00 & 1.00 & 0.00 & 0.00 & 9.29 \\
\hline Weish17 & 8633 & 1.00 & 0.00 & 1.00 & 0.00 & 1.00 & 0.00 & 0.00 & 9.17 \\
\hline Weish18 & 9580 & 1.00 & 0.00 & 1.00 & 0.00 & 1.00 & 0.00 & 0.00 & 10.86 \\
\hline Weish19 & 7698 & 1.00 & 0.00 & 1.00 & 0.00 & 1.00 & 0.00 & 0.00 & 13.56 \\
\hline Weish20 & 9450 & 1.00 & 0.00 & 0.10 & 0.00 & 1.00 & 0.00 & 0.00 & 10.96 \\
\hline Weish 21 & 9074 & 1.00 & 0.00 & 1.00 & 0.00 & 1.00 & 0.00 & 0.00 & 12.08 \\
\hline Weish 22 & 8947 & 1.00 & 0.00 & 1.00 & 0.00 & 1.00 & 0.00 & 0.00 & 12.72 \\
\hline Weish23 & 8344 & 1.00 & 0.00 & 1.00 & 0.00 & 1.00 & 0.00 & 0.00 & 12.83 \\
\hline Weish24 & 10220 & 1.00 & 0.00 & 1.00 & 0.00 & 1.00 & 0.00 & $\mathbf{0 . 0 0}$ & 12.61 \\
\hline Weish25 & 9939 & 1.00 & 0.00 & 1.00 & 0.00 & 1.00 & 0.00 & 0.00 & 12.67 \\
\hline Weish26 & 9584 & 1.00 & 0.00 & 1.00 & 0.21 & 1.00 & 0.00 & 0.00 & 14.39 \\
\hline Weish27 & 9819 & 1.00 & 0.00 & 1.00 & 0.00 & 1.00 & 0.00 & 0.00 & 14.40 \\
\hline Weish 28 & 9492 & 1.00 & 0.00 & 1.00 & 0.99 & 1.00 & 0.00 & 0.00 & 14.59 \\
\hline Weish29 & 9410 & 1.00 & 0.00 & - & - & 1.00 & 0.00 & 0.00 & 14.47 \\
\hline Weish30 & 11191 & 1.00 & 0.00 & 1.00 & 0.00 & 1.00 & 0.00 & 0.00 & 14.31 \\
\hline \#Best & & & 46 & 35 & 35 & 53 & 54 & 54 & \\
\hline$p$-value & & 0.564 & & 0.180 & 0.046 & & & & \\
\hline
\end{tabular}

TABLE XI

COMPARISONS OF TR-BDS, BCSA AND MA/PL (VS. TEST SET III)

\begin{tabular}{llllllll}
\hline \hline & TR-BDS & BCSA & \multicolumn{3}{c}{ MA/PL } \\
\hline & $A V G$ & $S D$ & $B E S T$ & $A V G$ & $B E S T$ & $A V G$ & $S D$ \\
\#Best & 16 & 15 & 48 & 34 & $\mathbf{5 4}$ & $\mathbf{5 3}$ & $\mathbf{5 3}$ \\
$p$-value & 0.000 & & 1.000 & 0.000 & & & \\
\hline \hline
\end{tabular}

4) Computational results for Test set IV

Results concerned with Test set IV are given in Tables XII to XIV. In Table XII, the $A V G$ values of MA/PL are better than those of other algorithms. Meanwhile, the $S D$ values of MA/PL are no worse than existing algorithms and are quite acceptable. In Tables XIII to XIV, the BEST and $A V G$ values of MA/PL are better than those of other algorithms for almost all the instances.
Moreover, the CPU (s) values of MA/PL are less than $220 \mathrm{~s}$, indicating the efficiency of MA/PL for large-sized instances.

TABLE XII

COMPARISONS OF KMTR-CUCKOO, KMTR-BH AND MA/PL (VS. TEST SET IV)

\begin{tabular}{|c|c|c|c|c|c|c|c|}
\hline \multirow{2}{*}{ nstance } & \multicolumn{2}{|c|}{ KMTR-Cuckoo } & \multicolumn{2}{|l|}{ KMTR-BH } & \multicolumn{3}{|l|}{ MA/PL } \\
\hline & $A V G$ & $S D$ & $A V G$ & $S D$ & $A V G$ & $S D$ & $\mathrm{U}$ \\
\hline & 1200 & 25.5 & 1200 & 25.3 & 12000 & 41.35 & 52.05 \\
\hline & & 57 & & & & & \\
\hline & & 39.8 & 7.9 & 50.3 & & 31.09 & .0 \\
\hline & & 32.1 & & & & & \\
\hline & & & & & & & \\
\hline & 4.6 & 35.4 & & 52.2 & 1219 & 46.72 & 45.91 \\
\hline & & 40. & & & & & \\
\hline & & 43.0 & & & & & \\
\hline & & 54.5 & & 91.3 & & 3.55 & 5.24 \\
\hline & & 62.2 & & & & & \\
\hline & & 47.8 & & 50.7 & & 40.72 & \\
\hline & & 63.9 & & & & & \\
\hline & & 53.3 & & & & & \\
\hline & & 45.4 & & 32.8 & & 6 & \\
\hline & & 49.0 & & & & & \\
\hline & & 40.8 & & 56.6 & & 6.77 & \\
\hline & & 47.2 & & & & 1 & \\
\hline & & 55.4 & & & & & \\
\hline & & 39 & & 41.2 & & 47.45 & \\
\hline & & 54 & & & & & \\
\hline & & & & & & & \\
\hline & & 59.1 & & & & & \\
\hline & & & & & & & \\
\hline & & 17 & & & & & \\
\hline & & 16 & & & & 27 & \\
\hline & & 24 & & & & & \\
\hline & & & & 37.9 & & & \\
\hline & & & & & & & \\
\hline & & 32 & & & & & \\
\hline & & 45 & & 34.1 & & 35.72 & .23 \\
\hline & 3 & 12 & & 1 & 22 & 1 & \\
\hline & & & & & & & \\
\hline
\end{tabular}

TABLE XIII

COMPARISONS OF KMTR-CUCKOO, KMTR-BH AND MA/PL (vS. TEST SET IV) \begin{tabular}{lllllll}
\hline \hline \multirow{2}{*}{ Instance } & \multicolumn{2}{l}{ KMTR-Cuckoo } & \multicolumn{2}{l}{ KMTR-BH } & MA/PL & \\
\cline { 2 - 4 } & $B E S T \quad A V G$ & $B E S T$ & $A V G$ & $B E S T$ & $A V G$ & CPU
\end{tabular} $\begin{array}{llllllllll}10.500 .00 & 117509 & 117302.8 & 117558 & 117293.0 & \mathbf{1 1 7 6 2 8} & \mathbf{1 1 7 5 7 1 . 2} & 159.11\end{array}$ $\begin{array}{llllllllll}10.500 .01 & 119072 & 118936.2 & \mathbf{1 1 9 2 3 2} & \mathbf{1 1 8 9 8 0 . 8} & 118974 & 118928.9 & 153.88\end{array}$ $\begin{array}{llllllllll}10.500 .02 & 119039 & 118723.0 & 118940 & 118840.5 & \mathbf{1 1 9 0 5 5} & \mathbf{1 1 8 9 5 0 . 4} & 166.51\end{array}$ $\begin{array}{lllllllll}10.500 .03 & 118586 & 118433.9 & 118598 & 118516.2 & \mathbf{1 1 8 6 8 0} & \mathbf{1 1 8 6 1 4 . 6} & 154.94\end{array}$ $\begin{array}{lllllllll}10.500 .04 & \mathbf{1 1 6 3 1 2} & 116013.8 & 116186 & 116095.5 & 116289 & \mathbf{1 1 6 2 2 9 . 6} & 158.44\end{array}$ $\begin{array}{llllllllll}10.500 .05 & 119257 & 119065.7 & 119257 & 119113.2 & 119318 & \mathbf{1 1 9 2 4 8 . 2} & 148.38\end{array}$ $\begin{array}{lllllllll}10.500 .06 & 119663 & 119506.6 & \mathbf{1 1 9 6 9 1} & 119556.7 & 119658 & \mathbf{1 1 9 5 6 6 . 1} & 163.87\end{array}$ $\begin{array}{llllllllll}10.500 .07 & 118058 & 117760.3 & 118016 & 117907.3 & \mathbf{1 1 8 1 2 1} & \mathbf{1 1 8 0 3 6 . 5} & 157.29\end{array}$ $\begin{array}{llllllllll}10.500 .08 & 117550 & 117235.0 & 117550 & 117363.0 & \mathbf{1 1 7 6 3 4} & \mathbf{1 1 7 5 4 6 . 6} & 162.53\end{array}$ $\begin{array}{llllllllll}10.500 .09 & 118962 & 118514.2 & 118896 & 118739.0 & \mathbf{1 1 9 0 8 6} & \mathbf{1 1 8 9 8 1 . 1} & 150.97\end{array}$ $\begin{array}{lllllllll}10.500 .10 & 217126 & 216892.0 & 216990 & 216892.0 & \mathbf{2 1 7 1 9 2} & \mathbf{2 1 7 1 2 5 . 7} & 135.13\end{array}$ $\begin{array}{lllllllll}10.500 .11 & 218872 & 218592.4 & 218672 & 218592.0 & \mathbf{2 1 8 9 2 2} & \mathbf{2 1 8 8 6 1 . 7} & 137.63\end{array}$ $\begin{array}{lllllllll}10.500 .12 & 217573 & 217542.3 & 217447 & 217358.8 & \mathbf{2 1 7 7 2 9} & \mathbf{2 1 7 6 2 1 . 9} & 115.63\end{array}$ $\begin{array}{lllllllll}10.500 .13 & 216570 & 216469.9 & 216570 & 216484.5 & \mathbf{2 1 6 7 1 1} & \mathbf{2 1 6 6 5 4 . 5} & 108.38\end{array}$ $\begin{array}{lllllllll}10.500 .14 & 213474 & 213363.5 & 213474 & 213374.0 & \mathbf{2 1 3 7 1 2} & \mathbf{2 1 3 6 1 9 . 1} & 144.96\end{array}$ $\begin{array}{lllllllll}10.500 .15 & 214829 & 214702.6 & 214761 & 214638.7 & \mathbf{2 1 4 8 8 7} & \mathbf{2 1 4 7 2 1 . 2} & 143.06\end{array}$ $\begin{array}{lllllllll}10.500 .16 & 217629 & 217567.1 & 217583 & 217484.2 & \mathbf{2 1 7 8 1 2} & \mathbf{2 1 7 7 2 4 . 1} & 146.39\end{array}$ $\begin{array}{lllllllll}10.500 .17 & 219675 & 219554.4 & 219589 & 219496.8 & \mathbf{2 1 9 8 1 4} & \mathbf{2 1 9 7 4 0 . 6} & 156.26\end{array}$ $\begin{array}{lllllllll}10.500 .18 & 214045 & 213939.4 & 214015 & 213862.7 & \mathbf{2 1 4 1 9 5} & \mathbf{2 1 4 0 9 7 . 0} & 151.19\end{array}$ $\begin{array}{lllllllll}10.500 .19 & 220582 & 220515.1 & 220488 & 220391.3 & \mathbf{2 2 0 7 5 2} & \mathbf{2 2 0 6 8 8 . 8} & 144.86\end{array}$ $\begin{array}{llllllllll}10.500 .20 & 304116 & 304019.1 & 304102 & 303991.6 & \mathbf{3 0 4 2 3 4} & \mathbf{3 0 4 1 3 2 . 7} & 114.55\end{array}$ $\begin{array}{llllllllll}10.500 .21 & 302263 & 302156.3 & 302138 & 302078.2 & \mathbf{3 0 2 2 6 5} & \mathbf{3 0 2 1 6 7 . 0} & 122.66\end{array}$ $\begin{array}{llllllllll}10.500 .22 & 302118 & 302061.6 & 302103 & 301968.2 & \mathbf{3 0 2 3 0 1} & \mathbf{3 0 2 1 9 5 . 8} & 134.62\end{array}$ $\begin{array}{lllllllll}10.500 .23 & 300566 & 300498.3 & 300542 & 300480.4 & 300665 & 300606.4 & 122.49\end{array}$ $\begin{array}{lllllllll}10.500 .24 & 304229 & \mathbf{3 0 4 1 8 7 . 7} & \mathbf{3 0 4 2 6 7} & 304168.7 & 304239 & 304147.7 & 115.83\end{array}$ $\begin{array}{lllllllll}10.500 .25 & 301445 & 301332.1 & \mathbf{3 0 1 7 3 0} & 301461.8 & 301621 & \mathbf{3 0 1 5 5 9 . 3} & 131.48\end{array}$ $\begin{array}{lllllllll}10.500 .26 & \mathbf{3 0 4 9 0 5} & \mathbf{3 0 4 8 1 4 . 6} & 304833 & 304778.1 & 304785 & 304689.3 & 107.56\end{array}$ $\begin{array}{llllllllll}10.500 .27 & \mathbf{2 9 6 3 6 1} & \mathbf{2 9 6 2 8 8 . 9} & 296263 & 296194.0 & 296337 & 296227.1 & 104.04\end{array}$ $\begin{array}{lllllllll}10.500 .28 & 301085 & 301031.2 & 301085 & 301026.0 & \mathbf{3 0 1 2 2 6} & \mathbf{3 0 1 1 4 9 . 1} & 116.26\end{array}$ $\begin{array}{lllllllll}10.500 .29 & 306881 & 306786.6 & 306881 & 306786.6 & \mathbf{3 0 6 9 7 3} & \mathbf{3 0 6 8 3 4 . 6} & 106.26\end{array}$ \begin{tabular}{lllllll} 
\#Best & 3 & 3 & 4 & 1 & $\mathbf{2 3}$ & $\mathbf{2 6}$ \\
$p$-value & 0.000 & 0.000 & 0.000 & 0.000 & & \\
\hline
\end{tabular} 
TABLE XIV

COMPARISONS OF KMTR-CUCKOO, KMTR-BH AND MA/PL (VS. TEST SET IV)

\begin{tabular}{llllllll}
\hline \hline \multirow{2}{*}{ Instance } & \multicolumn{2}{l}{ KMTR-Cuckoo } & \multicolumn{2}{l}{ KMTR-BH } & MA/PL & \\
\cline { 2 - 7 } & $B E S T$ & $A V G$ & $B E S T$ & $A V G$ & $B E S T$ & $A V G$ & CPU \\
\hline
\end{tabular}

\begin{tabular}{llllllllll}
\hline 30.500 .00 & 115526 & 115341.7 & 115449 & 115236.6 & $\mathbf{1 1 5 6 0 9}$ & $\mathbf{1 1 5 4 8 0 . 7}$ & 214.65
\end{tabular}

$\begin{array}{lllllllll}30.500 .01 & 114405 & 114291.5 & 114352 & 114172.2 & \mathbf{1 1 4 4 2 6} & \mathbf{1 1 4 2 9 9 . 9} & 214.96\end{array}$

$\begin{array}{lllllllll}30.500 .02 & 116256 & 116093.8 & 116158 & 116065.5 & \mathbf{1 1 6 3 5 7} & \mathbf{1 1 6 2 3 1 . 3} & 215.11\end{array}$

$\begin{array}{llllllllll}30.500 .03 & 114782 & 114673.9 & 114739 & 114574.4 & 114933 & 114815.9 & 199.56\end{array}$

$\begin{array}{llllllllll}30.500 .04 & 115995 & 115872.7 & 115994 & 115881.2 & \mathbf{1 1 6 1 4 6} & \mathbf{1 1 6 0 2 7 . 4} & 215.82\end{array}$

$\begin{array}{lllllllll}30.500 .05 & \mathbf{1 1 5 3 4 2} & 115143.0 & 115244 & 115149.5 & 115331 & \mathbf{1 1 5 1 5 0 . 6} & 216.66\end{array}$

$\begin{array}{lllllllll}30.500 .06 & \mathbf{1 1 3 7 1 2} & \mathbf{1 1 3 5 2 7 . 5} & 113593 & 113433.7 & 113621 & 113524.5 & 209.25\end{array}$

$\begin{array}{llllllllll}30.500 .07 & 113626 & 113516.5 & 113610 & 113522.0 & \mathbf{1 1 3 8 5 0} & \mathbf{1 1 3 6 8 3 . 7} & 195.16\end{array}$

$\begin{array}{lllllllll}30.500 .08 & 114822 & 114633.4 & 114705 & 114684.2 & \mathbf{1 1 5 0 4 9} & \mathbf{1 1 4 8 8 9 . 3} & 199.12\end{array}$

$\begin{array}{lllllllll}30.500 .09 & 116467 & 116338.6 & 116382 & 116374.5 & \mathbf{1 1 6 7 2 4} & \mathbf{1 1 6 5 9 5 . 7} & 160.12\end{array}$

$\begin{array}{llllllllll}30.500 .10 & 217776 & 217619.2 & 217607 & 217574.8 & \mathbf{2 1 7 8 5 8} & \mathbf{2 1 7 7 9 2 . 2} & 167.67\end{array}$

$\begin{array}{lllllllll}30.500 .11 & 214110 & 214002.7 & 214110 & 214089.5 & \mathbf{2 1 4 3 2 3} & \mathbf{2 1 4 2 0 9 . 7} & 179.88\end{array}$

$\begin{array}{lllllllll}30.500 .12 & 215638 & 215494.8 & 215580 & 215506.5 & \mathbf{2 1 5 6 6 8} & \mathbf{2 1 5 5 3 9 . 3} & 184.60\end{array}$

$\begin{array}{llllllllll}30.500 .13 & 217301 & 217215.8 & 217201 & 217136.0 & \mathbf{2 1 7 5 8 2} & \mathbf{2 1 7 4 8 1 . 4} & 175.30\end{array}$

$\begin{array}{lllllllll}30.500 .14 & 215116 & 214992.6 & 215036 & 214974.7 & \mathbf{2 1 5 3 0 4} & \mathbf{2 1 5 2 0 6 . 6} & 160.30\end{array}$

$\begin{array}{llllllllll}30.500 .15 & 215408 & 215219.7 & 215326 & 215223.6 & \mathbf{2 1 5 6 0 8} & \mathbf{2 1 5 4 6 7 . 7} & 189.97\end{array}$

$\begin{array}{lllllllll}30.500 .16 & 215576 & 215486.9 & 215516 & 215449.1 & \mathbf{2 1 5 7 6 4} & \mathbf{2 1 5 6 2 6 . 3} & 168.66\end{array}$

$\begin{array}{lllllllll}30.500 .17 & 216057 & \mathbf{2 1 6 0 0 2 . 6} & 215999 & 215981.6 & \mathbf{2 1 6 1 5 7} & 216000.9 & 170.98\end{array}$

$\begin{array}{llllllllll}30.500 .18 & 217013 & 216886.2 & 216882 & 216867.8 & \mathbf{2 1 7 1 0 3} & \mathbf{2 1 6 9 8 7 . 2} & 164.31\end{array}$

$\begin{array}{llllllll}30.500 .19 & 214332 & 214127.3 & 214194 & 214127.3 & \mathbf{2 1 4 4 5 1} & \mathbf{2 1 4 3 4 7 . 6} & 160.09\end{array}$

$\begin{array}{lllllllll}30.500 .20 & 301343 & 301241.0 & 301343 & 301200.8 & \mathbf{3 0 1 4 8 2} & \mathbf{3 0 1 3 5 0 . 6} & 156.89\end{array}$

$\begin{array}{lllllllll}30.500 .21 & 299720 & 299579.9 & 299636 & 299556.8 & \mathbf{2 9 9 9 0 8} & \mathbf{2 9 9 7 4 8 . 6} & 155.93\end{array}$

$\begin{array}{lllllllll}30.500 .22 & 304852 & 304748.2 & 304850 & 304774.5 & \mathbf{3 0 4 9 1 2} & \mathbf{3 0 4 8 5 4 . 4} & 141.40\end{array}$

$\begin{array}{llllllllll}30.500 .23 & 301645 & 301583.2 & 301658 & 301536.7 & \mathbf{3 0 1 7 4 2} & \mathbf{3 0 1 6 2 9 . 4} & 152.39\end{array}$

$\begin{array}{lllllllll}30.500 .24 & 304186 & 304106.1 & 304186 & 304082.4 & \mathbf{3 0 4 2 7 1} & \mathbf{3 0 4 1 8 5 . 4} & 155.78\end{array}$

$\begin{array}{lllllllll}30.500 .25 & 296521 & 296420.5 & 296450 & 296413.0 & \mathbf{2 9 6 7 4 4} & \mathbf{2 9 6 6 5 8 . 8} & 132.81\end{array}$

$\begin{array}{llllllllll}30.500 .26 & 302941 & 302843.8 & 302917 & 302841.6 & \mathbf{3 0 3 1 4 1} & \mathbf{3 0 3 0 0 2 . 7} & 153.15\end{array}$

$\begin{array}{lllllllll}30.500 .27 & 306616 & 306451.3 & 306616 & 306450.7 & \mathbf{3 0 6 7 7 4} & \mathbf{3 0 6 6 7 4 . 1} & 146.96\end{array}$

$\begin{array}{lllllllll}30.500 .28 & 302791 & 302565.8 & 302636 & 302550.3 & \mathbf{3 0 2 9 6 7} & \mathbf{3 0 2 8 8 1 . 9} & 153.41\end{array}$

$\begin{array}{lllllllll}30.500 .29 & 300170 & 300063.8 & 300170 & 300061.5 & \mathbf{3 0 0 2 6 9} & \mathbf{3 0 0 2 0 5 . 0} & 149.02\end{array}$

\begin{tabular}{cllllll} 
\#Best & 2 & 2 & 0 & 0 & $\mathbf{2 8}$ & $\mathbf{2 8}$ \\
$p$-value & 0.000 & 0.000 & 0.000 & 0.000 & & \\
\hline
\end{tabular}

\begin{tabular}{cccccc}
$p$-value & 0.000 & 0.000 & 0.000 & 0.000 & \\
\hline
\end{tabular}

Based on the results in Table XII, we draw the violin plots of KMTR-Cuckoo, KMTR-BH and MA/PL in Fig. 14, in terms of Ave. Dev and $S D$, showing that MA/PL is better than the other two algorithms. Therefore, there would be a large potential to use MA/PL for real-life problems that can be modeled as MKP.
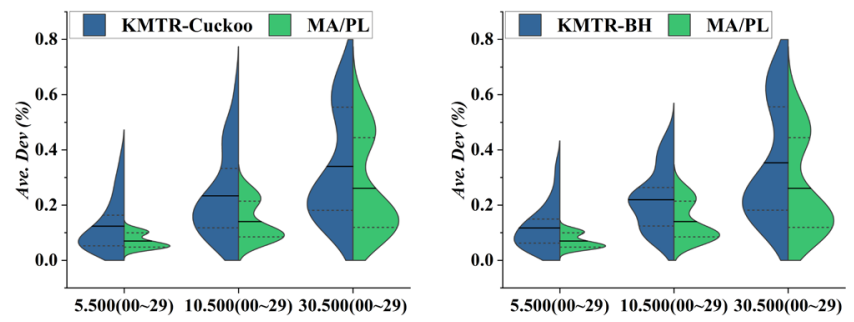

Fig. 14. Violin plots for KMTR-Cuckoo, KMTR-BH and MA/PL

\section{CASE Study}

Recently, the authors conducted a real-life project for an iron and steel company in Qian'an, Hebei, China. In this project, we focus on the production planning for the cold rolling of the iron and steel production process. Cold rolling is the most complex process in the iron and steel industry. The primary procedure of the cold rolling production planning is the order selection (OS), which involves selecting suitable orders (items) from a given order pool, on the basis of the capability of processing units (dimensions) and the due dates of orders. The sketch of OS is given in Fig. 15. We consider 5 bottleneck units in OS, i.e., acid pickling line (APL), pickling line tandem (PLT), continuous annealing 1 and 2 (CA1 and CA2) and twenty-high cold rolling mill (RCM). Note that in a given planning interval, each unit has a limited capacity (constraint) that is determined by the maintenance activity and the already assigned load.

To improve customer satisfaction, orders that are closer to their due dates should be given higher priority to be selected. We thus model OS as an MKP where the objective to be maximized is the total inverse of the time difference between the current time and the due date of each order. Besides, constraints considered here are the limited capacity of each unit in the current planning interval. In this section, OS is used as a case study and the related factory data are collected (available from http://dx.doi.org/10.13140/RG.2.2.21263.12964).

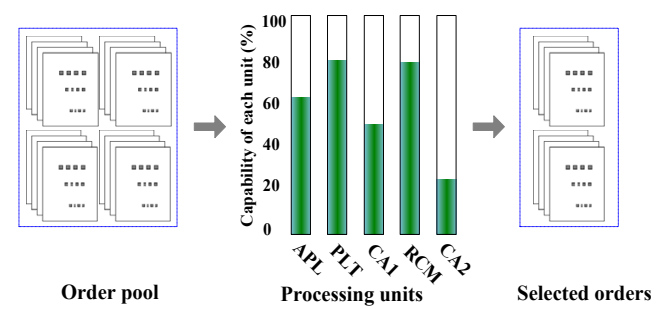

Fig. 15. Sketch of order selection in cold rolling process

In this case study, $\mathrm{MA} / \mathrm{PL}$ is independently run 30 times with the stopping condition genMax $=5000$. Besides, we specially invite two experienced schedulers (i.e., Schedulers A and B) from the company to manually make the decision of OS by using the same factory data as MA/PL. The results of MA/PL and the manual method are given in Table XV (details can be found at http://dx.doi.org/10.13140/RG.2.2.10777.36960).

TABLE XV

RESULTS OF MA/PL AND MANUAL METHOD (VS. CASE STUDY)

\begin{tabular}{llllll}
\hline \hline Decision method & $B E S T$ & $A V G$ & $S D$ & \#Selected order & CPU (s) \\
\hline MA/PL & 0.546 & 0.546 & 0.00 & 306 & 77.14 \\
Scheduler A & 0.520 & 0.520 & - & 270 & - \\
Scheduler B & 0.513 & 0.513 & - & 262 & - \\
\hline \hline
\end{tabular}

From Table $\mathrm{XV}$, it is clear that MA/PL outperforms, in terms of $B E S T$ and $A V G$, the manual method. Especially, MA/PL can select 306 orders with $S D=0.00$, which is much better than the two schedulers. Moreover, the value of CPU (s) of MA/PL is $77.14 \mathrm{~s}$, which is acceptable for the efficiency requirements of the company. Furthermore, we give the trend of objective found so far at each generation of MA/PL in Fig. 16, showing that MA/PL has a good convergence feature to solve OS. Toward that end, MA/PL can be seen as an effective and efficient solution method for practical applications.

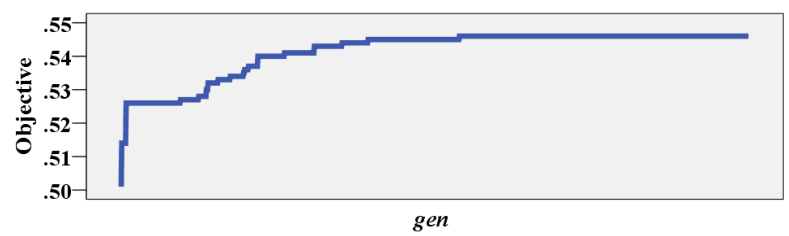

Fig. 16. Trend of objective at each generation of MA/PL (case study)

\section{CONCLUSIONS AND FUTURE WORK}

This paper proposed a memetic algorithm based on probability learning (i.e., MA/PL) to solve MKP. The primary high- 
lights of this work lie in two aspects, i.e., the problem-dependent heuristics and the learning-based search framework of MA/PL.

Based on the features of MKP, we proposed two problem-dependent heuristics for the repair operation and local search of MA/PL, i.e., RO_LUF and LS_LUF. These heuristics fully considered the tradeoff between the objective and the violation of constraints according to the presented LUFs. The framework of MA/PL adopted two kinds of probability distribution, i.e., MPD and JPD. MPD was concerned with the univariate probability distributions of items, whereas JPD focused on the bivariate dependency of conjoint items in a given spatial order. The learning scheme of MPD and JPD were all based on elite solutions recorded in ExA. To be specific, MPD was first estimated by the canonical competitive learning. And then JPD was constructed using the proposed learning rule based on a special binary Markov chain. By integrating and complementing MPD and JPD, more accurate probability models can be constructed accordingly. Moreover, we introduced a random sampling route based on the solution structures of MKP to reveal the overall dependency among all items by means of the bivariate dependency of two conjoint items. Thereby, promising offspring of MA/PL can be produced by the integration of MPD and JPD. Results of experiments and comparisons on 179 well-known benchmark instances and a real-life case study verified the effectiveness and practical values of the proposed MA/PL.

For further research, effective metaheuristics may be designed based on machine learning and mathematical programming to address other types of knapsack problems, such as the multi-objective multidimensional knapsack problem (MOMKP), the quadratic knapsack problem (QKP) and the multi-choice knapsack problem (MCKP).

\section{REFERENCES}

[1] S. Wei, "A branch and bound method for the multiconstraint zero-one knapsack problem,” J. Oper. Res. Soc., vol. 30, no. 4, pp. 369-378, 1979.

[2] P. C. Gilmore and R. E. Gomor, "The theory and computation of knapsack functions," Oper. Res., vol. 14, no. 6, pp. 1045-1074, 1966.

[3] Y. Shou, W. Xiang, Y. Li and W. Yao, "A multiagent evolutionary algorithm for the resource-constrained project portfolio selection and scheduling problem," Math. Probl. Eng., vol. 2014, no. 4, pp. 1-9, 2014.

[4] H. M. Weingartner and D. N. Ness, "Methods for the solution of the multidimensional 0/1 knapsack problem," Oper. Res., vol. 15, no. 1, pp. 83-103, 1967.

[5] B. Gavish and H. Pirkul, "Allocation of databases and processors in a distributed data processing," in: J. Akola (Ed.), Management of Distributed Data Processing, North-Holland, Amsterdam, pp. 215-231, 1982.

[6] P. C. Chu and J. E. Beasley, "A genetic algorithm for the multidimensional knapsack problem," J. Heuristics, vol. 4, no. 1, pp. 63-86, 1998.

[7] R. Breu and C. A. Burdet, "Branch and bound experiments in zero-one programming," Approaches to Integer Programming, in: Mathematical Programming Studies, vol. 2, pp. 1-50, Berlin: Springer Heidelberg, 2009.

[8] J. Puchinger, G. Raidl, R. Nther and U. Pferschy, "The multidimensional knapsack problem: structure and algorithms," INFORMS J. Comput., vol. 22, no. 2, pp. 250-265, 2010.

[9] R. Mansini and M. G. Speranza, "CORAL: An exact algorithm for the multidimensional knapsack problem," INFORMS J. Comput., vol. 24, no. 3, pp. 399-415, 2012.

[10]M. Gendreau and J. Y. Potvin, "Handbook of metaheuristics (Third Edition)," pp. 1-604, Springer, Cham, 2019.
[11]L. Tang, X. Wang and Z. Dong, "Adaptive multi-objective differential evolution with reference axis vicinity mechanism," IEEE T. Cybern, vol. 49, no. 9, pp. 3571-3585, 2018.

[12] X. Wang, Z. Dong and L. Tang, "A multi-objective differential evolution with personal archive and biased self-adaptive mutation selection," IEEE Trans. Syst. Man Cybern., 2018. (In Press)

[13]B. Qian, L. Wang, D. X. Huang and X. Wang, "Scheduling multi-objective job shops using a memetic algorithm based on differential evolution," Int. J. Adv. Manuf. Technol., vol. 35, pp. 1014-1027, 2008.

[14]J. Inführ and G. A. Raidl, "Memetic algorithm for the virtual network mapping problem," J. Heuristics, vol. 22, no. 4, pp. 475-505, 2016.

[15]R. Shang, K. Dai, L. Jiao and R. Stolkin, "Improved memetic algorithm based on route distance grouping for multiobjective large scale capacitated arc routing problems," IEEE T. Cybern., vol. 46, pp. 1000-1013, 2016.

[16]X. Wang and L. Tang, "A machine-learning based memetic algorithm for the multi-objective permutation flowshop scheduling problem," Comput. Oper. Res., vol. 79, pp. 60-77, 2017.

[17]E. Özcan and C. Başaran, "A case study of memetic algorithms for constraint optimization," Soft Comput., vol. 13, pp. 871-882, 2009.

[18]A. Rezoug, D. Boughaci and M. Badr-El-Den, "Memetic algorithm for solving the 0-1 multidimensional knapsack problem," $L N A I$, vol. 9273, pp. 298-304, 2015.

[19]K. Sörensen and M. Sevaux, "MAPM: memetic algorithms with population management," Comput. Oper. Res., vol. 33, pp. 1214-1225, 2004.

[20]A. S. Azad, M. M. Islam and S. Chakraborty, "A heuristic initialized stochastic memetic algorithm for MDPVRP with interdependent depot operations," IEEE T. Cybern., vol. 47, no. 12, pp. 4302-4315, 2007.

[21]Y. S. Ong and A. J. Keane, "Meta-Lamarckian learning in memetic algorithms," IEEE Trans. Evol. Comput., vol. 8, no. 2, pp. 99-110, 2004.

[22]P. Rakshit, A. Konar, P. Bhowmik, I. Goswami, S. Das, L. C. Jain and A. K. Nagar, "Realization of an adaptive memetic algorithm using differential evolution and Q-Learning: A Case Study in Multirobot Path Planning," IEEE Trans. Syst. Man Cybern. Part B, vol. 43, pp. 814-831, 2013.

[23]A. Gupta and Y. S. Ong, "Memetic computation: the mainspring of knowledge transfer in a data-driven optimization era," Springer, 2018.

[24]Q. H. Nguyen Q H, Y. S. Ong and M. H. Lim, "A probabilistic memetic framework," IEEE Trans. Evol. Comput., vol. 13, no. 3, pp. 604-623, 2009.

[25]Y. Liang, Z. Ren, X. Yao, Z. Feng, A. Chen and W. Guo, "Enhancing Gaussian estimation of distribution algorithm by exploiting evolution direction with archive," IEEE T. Cybern., vol. 50, no. 1, pp. 140-152, 2018.

[26]P. Yang, K. Tang and X. Lu, "Improving estimation of distribution algorithm on multimodal problems by detecting promising areas," IEEE T. Cybern., vol. 45, no. 8, pp. 1438-1449, 2014.

[27] Q. Yang, W. N. Chen, Y. Li, C. L. P. Chen, X. M. Xu and J. Zhang, "Multimodal estimation of distribution algorithms," IEEE T. Cybern., vol. 47, no. 3, pp. 636-650, 2016.

[28] V. A. Shim, K. C. Tan and C. Y. Cheong, "A hybrid estimation of distribution algorithm with decomposition for solving the multiobjective multiple traveling salesman problem," IEEE Trans. Syst. Man Cybern., vol. 42, no. 5, pp. 682-691, 2012.

[29]X. Ning and P. Li, "A cross-entropy approach to the single row facility layout problem," Int. J. Prod. Res., vol. 56, no. 11, pp. 3781-3794, 2018.

[30]R. Jovanovic, M. Tuba and S. Voß, "An efficient ant colony optimization algorithm for the blocks relocation problem,” Eur. J. Oper. Res., vol. 274, no. 1, pp. 78-90, 2019.

[31]X. Cai, H. Sun, Q. Zhang and Y. Huang, "A grid weighted sum Pareto local search for combinatorial multi and many-objective optimization," IEEE T. Cybern, vol. 49, no. 9, pp. 3586-3598, 2019.

[32]X. Cai, C. Xia, Q. Zhang, Z. Mei, H. Hu, L. Wang and J. Hu, "The collaborative local search based on dynamic-constrained decomposition with grids for combinatorial multiobjective optimization," IEEE T. Cybern, 2019. (In Press)

[33]F. Caraffini, A. V. Kononova and D. Corne, "Infeasibility and structural bias in differential evolution," Inf. Sci., vol. 496, pp. 161-179, 2019.

[34]L. Wang, S. Y. Wang and Y. Xu, "An effective hybrid EDA-based algorithm for solving multidimensional knapsack problem," Expert Syst. Appl., vol. 39, no. 5, pp. 5593-5599, 2012.

[35]Y. He, X. Zhang and W. Li, "An efficient binary differential evolution algorithm for the multidimensional knapsack problem," Eng. Comput., 2019. (In Press)

[36]P. Kumar, P. B. Barua and J. L. Gaindhar, "Quality optimization (multi-characteristics) through Taguchi's technique and utility concept," Qual. Reliab. Eng. Int., vol. 16, no. 6, pp. 475-485, 2015. 
[37] X. Chen, Y. S. Ong, M. H. Lim and K. C. Tan, "A multi-facet survey on memetic computation," IEEE Trans. Evol. Comput., vol. 15, no. 5, pp. 591-607, 2011.

[38] J. H. Lorie and L. J. Savage, "Three problems in rationing capital," Journal of Business, vol. 28, no. 4, pp. 229-239, 1955.

[39]H. M. Markowitz and A. S. Manne, "On the solution of discrete programming problems," Econometrica, vol. 25, no. 1, pp. 84-110, 1957.

[40]R. Loulou and E. Michaelides, "New greedy-like heuristics for the multidimensional 0-1 knapsack problem," Oper. Res., vol. 27, no. 6, pp. 1101-1114, 1979.

[41] M. J. Magazine and O. Oguz, "A fully polynomial approximation algorithm for the 0-1 knapsack problem," Eur. J. Oper. Res., vol. 8, no. 3, pp. 270-273, 1981.

[42]M. J. Magazine and O. Oguz, "A heuristic algorithm for the multidimensional zero-one knapsack problem," Eur. J. Oper. Res., vol. 16, no. 3, pp. 319-326, 1984.

[43] A. Fréville, "The multidimensional 0-1 knapsack problem: An overview," Eur. J. Oper. Res., vol. 155, no. 1, pp. 1-21, 2004.

[44] R. R. Hill, K. C. Yong and J. T. Moore, "Problem reduction heuristic for the 0-1 multidimensional knapsack problem," Comput. Oper. Res., vol. 39, no. 1, pp. 19-26, 2012.

[45]Y. Yoon, "A theoretical and empirical investigation on the Lagrangian capacities of the 0-1 multidimensional knapsack problem," Eur. J. Oper. Res., vol. 218, no. 2, pp. 366-376, 2012.

[46]A. Rong, K. Klamroth and J. R. Figueira, "Multicriteria 0-1 knapsack problems with $k$-min objectives," Comput. Oper. Res., vol. 40, no. 5, pp. 1481-1496, 2013.

[47] M. Chih, "Self-adaptive check and repair operator-based particle swarm optimization for the multidimensional knapsack problem," Appl. Soft. Comput., vol. 26, pp. 378-389, 2015.

[48]X. Zhang, C. Wu, J. Li, X. Wang, Z. Yang, J. M. Lee and K. H. Jung, "Binary artificial algae algorithm for multidimensional knapsack problems," Appl. Soft. Comput., vol. 43, pp. 583-595, 2016.

[49]D. Zouache, F. Nouioua and A. Moussaoui, "Quantum-inspired firefly algorithm with particle swarm optimization for discrete optimization problems," Soft Comput., vol. 20, no. 7, pp. 2781-2799, 2016.

[50] L. F. M. López, N. G. Blas and A. A. Albert, "Multidimensional knapsack problem optimization using a binary particle swarm model with genetic operations," Soft Comput., vol. 22, no. 8, pp. 2567-2582, 2018.

[51]K. K. Bhattacharjee and S. P. Sarmah, "Modified swarm intelligence based techniques for the knapsack problem," Appl. Intell., pp. 1-22, 2017.

[52] J. Liu, C. Wu, J. Cao, X. Wang and K. L. Teo, "A Binary differential search algorithm for the 0-1 multidimensional knapsack problem," Appl. Math. Model., vol. 40, no. 23-24, pp. 9788-9805, 2016.

[53] X. Kong, L. Gao, H. Ouyang and S. Li, "Solving large-scale multidimensional knapsack problems with a new binary harmony search algorithm," Comput. Oper. Res., vol. 63, pp. 7-22, 2015.

[54]J. García, B. Crawford, R. Soto, C. Castro and F. Paredes, "A $k$-means binarization framework applied to multidimensional knapsack problem," Appl. Intell., vol. 48, no. 2, pp. 357-380, 2018.

[55] A. Arin and G. Rabadi, "Integrating estimation of distribution algorithms versus Q-Learning into meta-raps for solving the 0-1 multidimensional knapsack problem," Comput. Ind. Eng., vol. 112, pp. 706-720, 2016.

[56] N. Rojas-Morales, M. C. Riff and U. E. Montero, "Learning from the opposite: Strategies for Ants that solve multidimensional Knapsack problem," in: Proc. CEC, IEEE, Vancouver, 2016.

[57] S. Baluja, "Population-based incremental learning: a method for integrating genetic search based function optimization and competitive learning," Technical Report CMU-CS-94-163, Carnegie Mellon University, 1994.

[58]Y. W. P. Hong and A. Scaglione, "Group testing for binary Markov sources: data-driven group queries for cooperative sensor networks," IEEE Trans. Inf. Theory, vol. 54, no. 8, pp. 3538-3551, 2008.

[59] ORLIB: http://people.brunel.ac.uk/ mastjjb/jeb/info.html.

[60]M. Abdel-Basset, D. El-Shahat, H. Faris and S. Mirjalili, "A binary multi-verse optimizer for 0-1 multidimensional knapsack problems with application in interactive multimedia systems," Comput. Ind. Eng., vol. 132, pp. 187-206, 2019.

[61]Z. Kern, Y. Lu and F. Vasko, "An OR practitioner's solution approach to the multidimensional knapsack problem," Int. J. Ind. Eng. Comput., vol. 11, no. 1, pp. 73-82, 2020.

[62] M. Chih, "Three pseudo-utility ratio-inspired particle swarm optimization with local search for multidimensional knapsack problem," Swarm Evol. Comput., vol. 39, pp. 279-296, 2018.

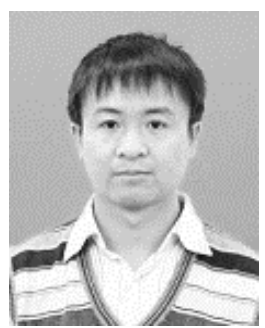

Zuocheng Li is currently working toward the Ph.D. degree in Key Laboratory of Data Analytics and Optimization for Smart Industry (Northeastern University), Ministry of China, Shenyang, China. His research interests include evolutionary algorithms, combinatorial optimization, machine learning and their applications.

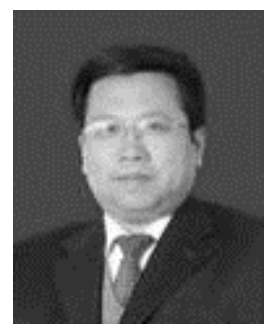

Lixin Tang (M'09-SM'14) received the B.Eng. degree in industrial automation, the M.Eng. degree in systems engineering and the Ph.D. degree in control theory and application from Northeastern University, Shenyang, China, in 1988, 1991, 1996, respectively.

He is a Fellow of Chinese Academy of Engineering, a Chair Professor of the Institute of Industrial \& Systems Engineering, Northeastern University; the Director of Key Laboratory of Data Analytics and Optimization for Smart Industry (Northeastern University), Ministry of Education, China; and the Head of Centre for Artificial Intelligence and Data Science.

His research interests cover industrial big data science, data analytics and machine learning, computational intelligent optimization, production and logistics batching and scheduling and engineering applications. His research papers have appeared in academic journals such as Operations Research, IIE Transactions, Naval Research Logistics, IEEE Transactions on Evolutionary Computation, IEEE Transactions on Power Systems, IEEE Transactions on Control Systems Technology, and European Journal of Operational Research.

He serves as an Associate Editor of IISE Transactions, IEEE Transactions on Evolutionary Computation, IEEE Transactions on Cybernetics, International Journal of Production Research, Journal of the Operational Research Society, in Editorial Board of Annals of Operations Research, and an Area Editor of Asia-Pacific Journal of Operational Research.

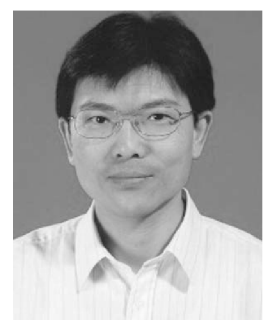

Jiyin Liu received the B.Eng. degree in industrial automation and the M. Eng. degree in systems engineering from Northeastern University, Shenyang, China, in 1982 and 1985, respectively, and the $\mathrm{Ph} . \mathrm{D}$. degree in manufacturing engineering and operations management from University of Nottingham, Nottingham, U.K., in 1993. He is a Professor of Operations Management with the School of Business and Economics, Loughborough University, Leicestershire, U.K. He is also a Cheung Kong Scholars Visiting Chair Professor with Institute of Industrial \& Systems Engineering, Northeastern University. His research interests include operations planning and scheduling problems in production; logistics and supply chains; and mathematical modeling, optimization, and heuristic methods. He has published papers in journals such as European Journal of Operational Research, IISE Transactions, International Journal of Production Research, IEEE Transactions on Evolutionary Computation, Naval Research Logistics, Operations Research, and Transportation Research. 